\title{
Overview of identification methods of mechanical parameters based on full-field measurements*
}

\author{
Stéphane AVRIL ${ }^{(a)}$ Marc Bonnet $^{(\mathrm{b}) \dagger}$ \Anne-Sophie BRETELLE $^{(\mathrm{c})}$, \\ Michel GRÉDIAC ${ }^{(\mathrm{d})}$, François HILD ${ }^{(\mathrm{e})}$, Patrick IENNY ${ }^{(\mathrm{c})}$, \\ Félix LATOURTE ${ }^{(\mathrm{f})}$, Didier Lemosse ${ }^{(\mathrm{g})}$, Stéphane PAGANO ${ }^{(\mathrm{f})}$, \\ Emmanuel PAGNACCO ${ }^{(\mathrm{g})}$, Fabrice PIERRON $^{(\mathrm{a})}$
}

Working group "Identification" of the French CNRS research network (GDR 2519) "Mesures de champs et identification en Mécanique des Solides / Full-field Measurements and Identification in Solid Mechanics".
(a) LMPF, ENSAM, Chalons en Champagne, France
(b) LMS, École Polytechnique, Palaiseau (Paris), France
(c) Ecole des Mines d'Alès, Alès, France
(d) Université Blaise Pascal, Clermont-Ferrand, France
(e) LMT, ENS Cachan, Cachan (Paris), France
(f) LMGC, Université Montpelier II, Montpellier, France
(g) LMR, INSA Rouen, Rouen, France

\begin{abstract}
This article reviews recently developed methods for constitutive parameter identification based on kinematic full-field measurements, namely the finite element model updating method (FEMU), the constitutive equation gap method (CEGM), the virtual fields method (VFM), the equilibrium gap method (EGM) and the reciprocity gap method (RGM). Their formulation and underlying principles are presented and discussed. These identification techniques are then applied to fullfield experimental data obtained on four different experiments, namely (i) a tensile test, (ii) the Brazilian test, (iii) a shear-flexural test, and (iv) a biaxial test. Test (iv) features a non-uniform damage field, and hence non-uniform equivalent elastic properties, while tests (i), (ii) and (iii) deal with the identification of uniform anisotropic elastic properties. Tests (ii), (iii) and (iv) involve non-uniform strain fields in the region of interest.
\end{abstract}

Keywords: Kinematic full-field measurements; Material identification; Finite element model

\section{Introduction}

The recent development of low-cost CCD cameras and the improvements in image processing with powerful and affordable PCs make non-contact measurement techniques more and more appealing in experimental mechanics $[78,80]$. Such techniques include digital image correlation, moiré and

\footnotetext{
*To appear in Experimental Mechanics (2008)

${ }^{\dagger}$ Corresponding author (bonnet@lms.polytechnique.fr)
} 
speckle interferometry, and grid methods. As a consequence, experimental procedures are gradually modified to take advantage of the large number of experimental data (e.g., typically 1,000 to 10,000 independent measurement points).

Using measurement of kinematic field variables such as displacements or strains for the identification of other mechanical parameters, most often associated with constitutive properties of the sample material, has become a very promising and active area of experimental mechanics. Compared to standard testing methods, where measured global quantities are used to infer the values of constitutive parameters with the help of simple exact solutions and under an assumption of homogeneous strain and stress fields in the zone of interest, full-field measurements allow considerably greater flexibility. They provide very rich experimental data when applied to tests conducted under non-homogeneous conditions (i.e. for which strains and stresses are not uniform in the zone of interest of the sample). Performing experiments of increased complexity thus allows to extract more (and more reliable) information from small numbers of tests. Moreover, larger sets of constitutive parameters may be identified, associated with e.g. anisotropic or non-homogeneous constitutive properties.

However, identification of parameters using this type of approach requires suitable computational strategies to analyze the experimental data. The aim of this article is to present an overview of recently-developed identification approaches specifically aimed at analyzing field measurements, and to demonstrate their application on experimental data obtained for several configurations. The exposition will focus on the case of quasi-static linear elasticity and small perturbations for the sake of simplicity. The identification of more complex constitutive properties is illustrated in one of the examples (identification of an isotropic damage field, Section 3.4) and commented upon in various parts of Section 2.

\subsection{Governing equations}

A generic elastic body whose undeformed configuration occupies the domain $\Omega$ is governed by three sets of equations, namely the equilibrium equations

$$
\begin{cases}\operatorname{div} \boldsymbol{\sigma}=\mathbf{0} & \text { in } \Omega \\ \boldsymbol{\sigma} \cdot \boldsymbol{n}=\overline{\boldsymbol{T}} & \text { on } S_{f}\end{cases}
$$

(having for simplicity assumed the absence of body forces) the kinematic compatibility equations

$$
\left\{\begin{array}{l}
\boldsymbol{\varepsilon}=\frac{1}{2}\left(\nabla \boldsymbol{u}+\nabla^{t} \boldsymbol{u}\right) \quad \text { in } \Omega, \\
\boldsymbol{u}=\overline{\boldsymbol{u}} \text { on } S_{u},
\end{array}\right.
$$

and the constitutive equation

$$
\boldsymbol{\sigma}=\mathcal{A}: \varepsilon \quad \text { in } \Omega
$$

In equations (1)-(3), $\boldsymbol{u}$ denotes the displacement vector, $\boldsymbol{\varepsilon}$ the infinitesimal strain tensor, $\boldsymbol{\sigma}$ the Cauchy stress tensor and $\boldsymbol{n}$ the outward unit normal vector. Overlined quantities are prescribed as static or kinematic boundary conditions. The surfaces $S_{u}$ and $S_{f}$ are such that $S_{u} \cup S_{f}=\partial \Omega$ and $S_{u} \cap S_{f}=\emptyset$, so as to support a well-posed set of boundary conditions. Components of the Hooke tensor $\mathcal{A}$ can be either constant (homogeneous material) or point-dependent (heterogeneous material). If the elastic properties are isotropic, they are described in terms of two independent scalar moduli, e.g. the Lamé constants $\lambda, \mu$.

It is customary and convenient to introduce the sets $\mathcal{S}$ and $\mathcal{C}$ of statically admissible stress fields and of kinematically admissible displacement fields, respectively, i.e.:

$$
\begin{aligned}
\mathcal{S} & =\left\{\boldsymbol{\tau} \in \mathcal{V}_{\sigma}, \operatorname{div} \boldsymbol{\tau}=\mathbf{0}(\text { in } \Omega) \text { and } \boldsymbol{\tau} \cdot \boldsymbol{n}=\overline{\boldsymbol{T}}\left(\text { on } S_{f}\right)\right\} \\
\mathcal{C}(\overline{\boldsymbol{u}}) & =\left\{\boldsymbol{v} \in \mathcal{V}_{u}, \boldsymbol{v}=\overline{\boldsymbol{u}} \quad\left(\text { on } S_{u}\right)\right\}
\end{aligned}
$$


where $\mathcal{V}_{\sigma}$ is the set of symmetric second-order tensor fields $\tau$ that are, together with $\operatorname{div} \tau$, squareintegrable over $\Omega$, and $\mathcal{V}_{u}$ is the set of vector (displacement) fields defined over $\Omega$ and having a bounded strain energy on $\Omega$.

\subsection{Direct problem}

The usual problem of solid mechanics consists of determining the displacement, strain and stress fields given the geometry of the solid, the constitutive parameters, and a well-posed set of boundary conditions. Such problems are usually referred to as direct in the literature. Equations (1-3) constitute a typical direct problem. Closed-form solutions to direct problems are available only in very particular cases. Hence numerical solution techniques are usually called for, the most commonly used being the finite element method (FEM).

The customary version of the FEM, employing displacement as the primary unknown, is considered here. It can be based upon the virtual work principle, which for equations (1)-(3) reads

$$
\int_{\Omega} \boldsymbol{\varepsilon}\left[\boldsymbol{u}^{\star}\right]: \mathcal{A}: \varepsilon[\boldsymbol{u}] \mathrm{d} V-\int_{S_{f}} \boldsymbol{u}^{\star} \cdot \boldsymbol{T} \mathrm{d} S=0 \quad \text { for all } \boldsymbol{u}^{\star} \in \mathcal{C}(\mathbf{0})
$$

The usual Galerkin discretization procedure using FEM shape functions provides the standard set of FEM equations

$$
\boldsymbol{K}(\mathcal{A}) \boldsymbol{U}=\boldsymbol{R}
$$

where $\boldsymbol{K}(\mathcal{A})$ is the stiffness matrix, here restricted to the non-prescribed degrees of freedom (DOFs), $\boldsymbol{R}$ is the generalized load vector which incorporates the prescribed loads (1b) and (2b), and the vector $\boldsymbol{U}$ gathers the unknown DOFs introduced by the FEM discretization (usually nodal displacements). The notation $\boldsymbol{K}(\mathcal{A})$ stresses the obvious but essential fact that the stiffness matrix depends on the constitutive parameters.

\subsection{Identification problem}

Constitutive parameter identification is often referred to as an inverse problem (see [9] for a recent review of inverse problems in elasticity). The lack of information about $\mathcal{A}$ as a tensor-valued function (for heterogeneous materials), or about elastic moduli entering the components of $\mathcal{A}$ (for homogeneous materials), must be compensated with overdetermined data. In other words, in addition to the boundary data $\overline{\boldsymbol{u}}$ and $\overline{\boldsymbol{T}}$ as stipulated in (1) and (2), supplementary information resulting from measurements must be available. Such additional information may take many forms, and is here assumed to consist of kinematic field measurements.

In many practical cases, the primary unknown of the identification problem is a vector $\boldsymbol{\theta}=$ $\left\{\theta_{1}, \ldots, \theta_{M}\right\}$ of $M$ constitutive parameters entering the elasticity tensor: $\mathcal{A}=\mathcal{A}(\boldsymbol{\theta})$, e.g. $\boldsymbol{\theta}=$ $(K, G)$ and $M=2$ for an isotropic elastic material. Of course, the stiffness matrix becomes also parameterized by $\boldsymbol{\theta}$ as a result: $\boldsymbol{K}(\mathcal{A})=\boldsymbol{K}(\mathcal{A}(\boldsymbol{\theta}))=\tilde{\boldsymbol{K}}(\boldsymbol{\theta})$, so that (7) reads

$$
\tilde{\boldsymbol{K}}(\boldsymbol{\theta}) \boldsymbol{U}=\boldsymbol{R}
$$

When the parameterization $\boldsymbol{A}(\boldsymbol{\theta})$ is linear in $\boldsymbol{\theta}$ (which is the case e.g. with $\boldsymbol{\theta}=(K, G)$ ), it is convenient to set $\mathcal{A}(\boldsymbol{\theta})$ and $\tilde{\boldsymbol{K}}(\boldsymbol{\theta})$ in the form

$$
\mathcal{A}=\sum_{i=1}^{M} \mathcal{A}_{i} \theta_{i}, \quad \tilde{\boldsymbol{K}}(\boldsymbol{\theta})=\boldsymbol{K}(\mathcal{A}(\boldsymbol{\theta}))=\sum_{i=1}^{M} \tilde{\boldsymbol{K}}_{i} \theta_{i}
$$


A frequent consideration is that only partial information on the applied loads (typically, values of load resultants) are available (i.e. the load distribution $\bar{T}$ in (1b) is not fully known). In such cases, the set $\mathcal{S}$ of admissible stresses is re-defined as

$$
\left.\mathcal{S}=\left\{\boldsymbol{\tau} \in \mathcal{V}_{\sigma}\right), \operatorname{div} \boldsymbol{\tau}=\mathbf{0} \text { in } \Omega, \ell_{1}(\boldsymbol{\tau} . \boldsymbol{n})=\boldsymbol{g}_{1}, \ldots, \ell_{N}(\boldsymbol{\tau} . \boldsymbol{n})=\boldsymbol{g}_{N}\right\}
$$

instead of (4), with each constraint $\ell_{k}(\boldsymbol{\tau} \cdot \boldsymbol{n})=\boldsymbol{g}_{k}$ expressing a force measurement, e.g.

$$
\ell_{k}(\boldsymbol{\tau} \cdot \boldsymbol{n})=\int_{\Gamma} \boldsymbol{\tau} \cdot \boldsymbol{n} \mathrm{d} S
$$

for a known load resultant on part $\Gamma$ of the boundary.

Closed-form solutions for constitutive parameter identification are available in some very particular cases only (e.g., uniaxial tensile test, 3-point or 4-point bending tests), for which the strain distribution is constant or linear and can be directly determined from Equation (1). The constitutive parameters are therefore directly related to the measured strain/displacement components and the applied forces. Most often, however, the strain/stress fields are heterogeneous and the link between the measured quantities and the constitutive parameters is implicit, through the set of governing equations $(1,2,3)$, and cannot easily be expressed in terms of explicit formulae. This article aims at reviewing methods recently proposed for parameter identification based on field measurements, namely:

1. The finite element model updating method (FEMU): conceptually the most intuitive approach, it consists in performing iteratively finite element simulations of the test so as to find constitutive parameters that achieve the best match between computed and actual measurements (section 2.1).

2. The constitutive equation gap method (CEGM), which stems from a more general approach initially developed for updating FEM models from vibrational data or assessing quality of FEM meshes. The minimization of a constitutive equation gap functional (of which many variants are available) provides the identified values of (possibly heterogeneous) constitutive parameters (section 2.2).

3. The virtual fields method (VFM), based on the virtual work principle applied with well-chosen virtual fields. It leads to explicit identification formulae in the case of linear elasticity. This approach requires full-field measurements in the domain (section 2.3).

4. The equilibrium gap method (EGM), based on the discretization of the equilibrium equations. This approach allows to determine heterogeneous elastic (or damage) fields by minimizing the equilibrium gap (section 2.4).

5. The reciprocity gap method (RGM), based on the Maxwell-Betti reciprocity theorem and adjoint fields. Constitutive properties are found by minimizing (or setting to zero) the reciprocity gap for any adjoint field. This approach requires full-field measurements on the boundary (section 2.5).

A presentation and discussion of the formulation and underlying principles of these methods (sections 2.1 to 2.5 ) is followed by results of their application to experimental data (section 3 ). 


\section{Overview of identification techniques using full-field data}

\subsection{The finite element model updating approach}

\subsubsection{Principle}

The goal of the finite element model updating (FEMU) approach is to identify the constitutive parameters $\boldsymbol{\theta}$ by exploiting equation (8), or similar equations stemming from a FEM model of the sample. Basically, the FEMU approach is based on minimizing the discrepancy between either known and predicted forces (FEMU-F), or measured and predicted displacement fields (FEMU-U). Both approaches are now summarized.

The force balance method (FEMU-F). The force balance method [21,74], also known as the input residual method or the equilibrium method, is based on the experimental availability of all the nodal displacements $\hat{\boldsymbol{U}}$ and prescibed forces $\hat{\boldsymbol{R}}$. Substituting the known quantities $\hat{\boldsymbol{U}}, \hat{\boldsymbol{R}}$ into (8) yields a set of equations used as a basis for recovering the constitutive unknowns $\boldsymbol{\theta}$. This set of equations is in practice verified only in an approximate sense only due to experimental, discretization and modelling errors. It is, moreover, usually overdetermined w.r.t. $\boldsymbol{\theta}$. Hence, the FEMU-F approach usually consists in minimizing a least-squares functional of the form

$$
J_{\mathrm{F}}(\boldsymbol{\theta})=\frac{1}{2}(\hat{\boldsymbol{R}}-\tilde{\boldsymbol{K}(\boldsymbol{\theta}) \hat{\boldsymbol{U}}})^{\mathrm{T}} \boldsymbol{W}_{\mathrm{F}}(\hat{\boldsymbol{R}}-\tilde{\boldsymbol{K}}(\boldsymbol{\theta}) \hat{\boldsymbol{U}}) .
$$

where $\boldsymbol{W}_{\mathrm{F}}$ is a (symmetric, positive definite) weighting matrix. When the parameterization $\mathcal{A}(\boldsymbol{\theta})$ is linear in $\boldsymbol{\theta}$, the minimization of $J_{\mathrm{F}}(\boldsymbol{\theta})$ leads to a linear system of equations in $\boldsymbol{\theta}$, e.g. (using the notations introduced in Eq. (9)) the normal equations

$$
\sum_{i=1}^{M}\left(\tilde{\boldsymbol{K}}_{j} \hat{\boldsymbol{U}}\right)^{\mathrm{T}} \boldsymbol{W}_{\mathrm{F}}\left(\tilde{\boldsymbol{K}}_{i} \hat{\boldsymbol{U}}\right) \theta_{i}=\left(\tilde{\boldsymbol{K}}_{j} \hat{\boldsymbol{U}}\right)^{\mathrm{T}} \boldsymbol{W}_{\mathrm{F}} \hat{\boldsymbol{R}} \quad(1 \leq j \leq M) .
$$

Another treatment is based on defining a well-chosen set $\boldsymbol{U}_{i}^{\star}$ of (FEM-discretized) virtual fields so as to have $\boldsymbol{U}_{i}^{\star}$ satisfy $\boldsymbol{U}^{\star \mathrm{T}} \tilde{\boldsymbol{K}}_{i} \hat{\boldsymbol{U}}=\boldsymbol{I}$. Each parameter $\theta_{i}$ is then obtained simply through

$$
\boldsymbol{\theta}_{i}=\boldsymbol{U}_{i}^{\star \mathrm{T}} \hat{\boldsymbol{R}}
$$

It is worth emphasizing that the input residual method does not require any direct FEM solution.

Displacement method. In many cases, the displacements are only partially known, and the force method cannot be applied. The displacement method [21,39,74], also known as the output residual method has hence been developed as an alternative approach. It consists in minimizing cost functions of the form

$$
J_{\mathrm{U}}(\boldsymbol{\theta} ; \varpi)=\frac{1}{2}(\hat{\boldsymbol{U}}-\boldsymbol{U}(\boldsymbol{\theta} ; \varpi))^{\mathrm{T}} \boldsymbol{W}_{\mathrm{U}}(\hat{\boldsymbol{U}}-\boldsymbol{U}(\boldsymbol{\theta} ; \varpi))
$$

where $\boldsymbol{U}(\boldsymbol{\theta} ; \varpi)$ is the solution to a well-posed direct elasticity problem, $\varpi$ symbolizes the available information on boundary conditions, and $\boldsymbol{W}_{\mathrm{U}}$ is a (symmetric, positive definite) weighting matrix. The computed displacement $\boldsymbol{U}(\boldsymbol{\theta} ; \boldsymbol{R})$ normally does not depend linearly on $\boldsymbol{\theta}$. The minimizer $(\boldsymbol{\theta}, \boldsymbol{R})$ of $J_{\mathrm{U}}(\boldsymbol{\theta}, \boldsymbol{R})$ subject to resulting-load constraints of form (11) is then sought by means of an iterative procedure. This approach can be given several forms according to the choice of boundary conditions. When the prescribed forces are either completely known or completely unknown, resulting-load constraints are not necessary and an unconstrained minimization is performed on $J_{U}(\boldsymbol{\theta}, \varpi)$. This approach will herein be referred to by the acronym FEMU-U. 
Another variation on this idea consists in applying a known load over the whole boundary [73] (i.e. with $S_{u}=\emptyset$ ). This entails a proper management of the spurious rigid-body modes affecting the direct solution, but the identified parameters are less sensitive to imperfectly-known displacements on the boundary.

Comments about the FEMU approach. Many variants of the above-defined formulations can be obtained by varying the matrix weights $\boldsymbol{W}_{\mathrm{F}}, \boldsymbol{W}_{\mathrm{U}}$ (for example, $J_{\mathrm{U}}(\boldsymbol{\theta})$ becomes homogeneous to an energy with $\boldsymbol{W}_{\mathrm{U}}=\boldsymbol{K}$ and is close to $J_{\mathrm{F}}$ with $\boldsymbol{W}_{\mathrm{U}}=\boldsymbol{K}^{2}$ ), or by combining $J_{\mathrm{F}}$ and $J_{\mathrm{U}}$ to form a mixed misfit function (a strategy [28, 46, 62] referred to herein as FEMU-U-F). The latter formulation is better suited to problems involving stresses and strains concentrations and localised damage. A misfit function involving mechanical strains has been proposed in [56]. More sophisticated misfit functions that incorporate prior information may be defined on the basis of Bayesian techniques [81].

Depending on the choice of measurable quantity being used for identification purposes, a very wide range of situations can be considered. In fact, it is desirable, but by no means necessary, that field measurements be used, because in principle the FEMU approach may be applied to any kind of overdetermined data, e.g. overdetermined data on the sample boundary. The output method is especially flexible with respect to accommodating many kinds of incomplete data, whereas the basic design of the input method requires full-field measurements. Using the latter in other cases entails model condensation or data expansion strategies (based on the numerical model).

Minimisation of $J_{\mathrm{F}}$ or $J_{\mathrm{U}}$ is often carried out by Gauss-Newton or Levenberg-Marquardt methods (see examples in e.g. [28] and [74], respectively). Such methods are relatively economical in terms of overall number of FEM solutions required, and usually adequate for problems with moderate numbers of unknown constitutive parameters. Global optimization methods (e.g. evolutionary algorithms, see [12] for an overview), which entail large numbers of FEM solutions, may be called for in situations involving large numbers of unknown parameters, e.g. identification of heterogeneous material characteristics.

Finally, the direct solution $\boldsymbol{U}(\boldsymbol{\theta})$ may be set up using methods other than FEM, e.g. the finite difference method [82], the boundary element method [8], or (occasionally) closed-form solutions [45].

\subsubsection{Applications to full-field measurements}

The FEMU methodology is a priori suited for a wide range of applications, featuring complex geometrical and loading configurations. Early studies exploiting FEMU approaches include [52] (statics) and [19] (free vibrations). These studies exploit only a low number of measured displacements or vibrational eigenfrequencies, and as a result often lead to undetermined identification problems. With the subsequent progress of full-field measurement techniques, material properties are often identified on the basis of the complete field data. Following is an overview of such applications.

Linear elasticity. References $[56,57,68]$ address the identification of the four elasticity parameters for orthotropic planar elasticity from uniaxial test measurements on a specimen having a hole in its center. In [12], the elastic properties of a human basin are identified from surface measurements using a 3-D FEM model featuring two homogeneous materials. Data from modal analyses is used in [22]

Bending rigidities of thin anisotropic plates. Several studies concern the identification of the bending rigidities of thin anisotropic plates, e.g. [63] where deflection fields at the surface of bent composite and cardboard plates are measured using a shadow moiré technique. A FEMU-U-based parameter identification turned out to be unsuccessful because the test configuration used, based on 4-point plate bending, was not sensitive to all rigidities. To overcome this difficulty and ensure that 
the measurements are sensitive to all rigidities, a test design approach based on finite element sensitivities was subsequently developed [2,3]. This investigation also showed that a formulation of the cost function in terms of curvatures instead of deflections gave better results, although this benefit would likely disappear for experimental curvatures obtained by a second-order numerical differentiation of measured deflections. Unfortunately, no experimental applications were performed in this study.

Viscoelasticity. More recently, experimental implementation of bending tests has been performed on structural wood-based panels [61], using test configurations designed using the procedure of [2,3]. The optical technique provided the deflection field by means of fringe projection. The cost function used to identify the elastic rigidities is of type (15). For viscoelastic parameters, measurements are taken at several times (creep test) and used in the output residual method. This study yielded satisfactory values for the elastic parameters, but only preliminary results for the viscoelastic parameters, which were not compared to reference values.

In addition, vibroacoustic treatments involve new visco-elastic materials whose characterisation is necessary for subsequent use in FE models. Frequency-dependent complex moduli are recovered in $[69,75]$ by means of a FEMU-F approach using measured frequency-response displacement fields, and in [75].

In-plane elasto-plastic or viscoplastic behaviour of metals. FEMU has also been applied to the identification of parameters driving the elasto-plastic behaviour of metals [65,66], with promising results, from tensile tests on notched or drilled specimens giving rise to heterogeneous stress states for which displacements were measured using digital image correlation (DIC) and the total tensile force was known. Various constitutive models have been tested (with linear or non linear hardening). The cost function is of type (15), with $\boldsymbol{W}_{\mathrm{U}}$ chosen according to the Gauss-Markov estimator. The boundary conditions of the finite element model are provided by the DIC patterns located at the boundary of the measurement zone (near the clamps).

Large strain elastoplasticity is considered in [50], where material parameters are estimated for a piecewise-linear plasticity model and a parabolic hardening model. Viscoplastic constitutive parameters are subsequently identified from a high strain-rate tensile test in [51].

Hyperelasticity. Parameter identification for an hyperelastic constitutive law (Mooney-Rivlin) from biaxial tests is considered in [24]. In [28], the same constitutive model is considered for a PET specimen under uniaxial test in a FEMU-F framework.

Other examples. The group of C.W.J. Oomens at Eindhoven University of Technology has undertaken a number of applications of such approaches in the field of biomechanics. For instance, a point tracking video system was used to characterize the anisotropic and non-linear behaviour of human skin in vivo [64]. The test setup is directly mounted onto a person's arm and $12 \times 6$ markers are used to follow the deformation of the skin. Another example, again based on the point tracking system, concerns the characterization of the orthotropic behaviour of dog skin in vitro from biaxial tests [71, 84]. Here, orthotropy directions vary spatially because of the arrangement of collagen fibres.

Other applications concern the identification of boundary conditions, see e.g. [72], or [74] where boundary conditions and elastic parameters are simultaneously identified from measured displacement field and force resultant applied to the specimen. 


\subsection{The constitutive equation gap method}

The constitutive equation gap measures the distance between a given stress field $\tau$ and another stress field computed through a constitutive model from a given displacement field $\boldsymbol{v}$. If the constitutive model is linearly elastic, then the constitutive equation gap (CEG) between the fields $\boldsymbol{\tau}$ and $\boldsymbol{v}$ is defined by

$$
\mathcal{E}(\boldsymbol{v}, \boldsymbol{\tau}, \mathcal{A})=\frac{1}{2} \int_{\Omega}(\boldsymbol{\tau}-\mathcal{A}: \varepsilon[\boldsymbol{v}]): \mathcal{A}^{-1}:(\boldsymbol{\tau}-\mathcal{A}: \varepsilon[\boldsymbol{v}]) \mathrm{d} V
$$

where the elasticity tensor $\mathcal{A}$ is allowed to be heterogeneous (note that the presence of the compliance tensor $\mathcal{A}^{-1}$ in the integrand confers the physical dimension of an energy to $\mathcal{E}(\boldsymbol{v}, \boldsymbol{\tau}, \mathcal{A})$ ). The following remarks explain the usefulness of the concept of CEG:

(i) The solution $(\boldsymbol{u}, \boldsymbol{\sigma})$ to a usual well-posed boundary value problem of linear elasticity, such as that defined by equations $(1,2,3)$, is characterized by

$$
(\boldsymbol{u}, \boldsymbol{\sigma})=\underset{(v, \tau) \in \mathcal{C}(\bar{u}) \times \mathcal{S}}{\arg \min } \mathcal{E}(\boldsymbol{v}, \boldsymbol{\tau}, \mathcal{A}) \quad \text { and } \quad \mathcal{E}(\boldsymbol{u}, \boldsymbol{\sigma}, \mathcal{A})=0
$$

where $\mathcal{S}$ and $\mathcal{C}(\overline{\boldsymbol{u}})$ are the sets of admissible stresses and displacements defined by (4) and (5).

(ii) Considering a constitutive parameter identification problem, where for instance the elasticity tensor $\mathcal{A}$ is to be identified, one may alter definitions (4) and (5) of the sets of admissible fields so as to include all available experimental information about displacements and stresses. Based on such adjusted definitions, $\mathcal{A}$ can be sought so as to minimize the constitutive equation gap:

$$
\mathcal{A}=\underset{\mathcal{A}^{\star} \in \mathbb{A}}{\arg \min } J\left(\mathcal{A}^{\star}\right) \quad \text { with } \quad J\left(\mathcal{A}^{\star}\right)=\min _{(v, \tau) \in \mathcal{C} \times \mathcal{S}} \mathcal{E}\left(\boldsymbol{v}, \boldsymbol{\tau}, \mathcal{A}^{\star}\right)
$$

where $\mathbb{A}$ is the set of admissible elasticity tensor fields, i.e. of fourth-order tensor functions $\mathcal{A}(\boldsymbol{x})$ that fulfill the symmetry, positive definiteness and coercivity properties expected of any linear elastic constitutive relation.

Equation (18) defines the essence of the constitutive equation gap method (CEGM). In particular, the procedure is seen to involve an alternate-direction search where a partial minimization with respect to $(\boldsymbol{v}, \boldsymbol{\tau})$ is followed by a partial minimization with respect to $\mathcal{A}^{\star}$.

The CEGM is in principle applicable to any identification problem where overdetermined data is available, i.e. the overdetermined data does not need to be a full-field measurement. Indeed, this approach has been extensively used in the context of model updating from vibrational data [53,54]. On the other hand, the CEGM framework accommodates full-field measurements in a very natural way. Two variants of the CEGM, applicable to the identification of heterogeneous elasticity tensors, are now summarized. For both, a measurement $\hat{\boldsymbol{u}}$ of the displacement $\boldsymbol{u}$ is assumed to be available.

First variant: exact enforcement of kinematic measurements. In this variant, the measured field $\hat{\boldsymbol{u}}$ is introduced directly into the CEG functional, and $\mathcal{A}$ is sought by means of the minimization problem

$$
\mathcal{A}=\underset{\mathcal{A}^{\star} \in \mathbb{A}}{\arg \min } J\left(\mathcal{A}^{\star}\right) \quad \text { with } \quad J\left(\mathcal{A}^{\star}\right)=\min _{\tau \in \mathcal{S}} \mathcal{E}\left(\hat{\boldsymbol{u}}, \boldsymbol{\tau}, \mathcal{A}^{\star}\right)
$$

where the set $\mathcal{S}$ of admissible stresses is defined by (10) to accommodate force-measurement constraints. The minimization (19b) is performed by means of a finite element interpolation of admissible stress fields using e.g. Q1 elements for plane problems. Enforcing the equilibrium and forcemeasurement constraints entered in the definition (10) of $\mathcal{S}$ by means of Lagrange multipliers, the 
variational problem

$$
\inf _{\boldsymbol{\tau} \in H(\operatorname{div})} \sup _{\gamma, \lambda_{i}} \mathcal{E}\left(\hat{\boldsymbol{u}}, \boldsymbol{\tau}, \mathcal{A}^{\star}\right)+\gamma_{1} \int_{\Omega} \boldsymbol{\gamma} \cdot \operatorname{div} \boldsymbol{\tau} \mathrm{d} V+\sum_{i} \lambda_{i}\left(\ell_{i}(\boldsymbol{\tau} \cdot \boldsymbol{n})-\boldsymbol{g}_{i}\right)
$$

is numerically solved. The subsequent minimization of $J\left(\mathcal{A}^{\star}\right)$ is explicit.

With this approach, at most three material coefficients can be locally determined (e.g. treating coefficients as constant over each finite elements or over predefined subsets of finite elements). Consequently, it is natural to formulate the approach in terms of elastic models with cubic symmetry and the $Q_{x x} Q_{y y} Q_{s s}$ coefficients of $\mathcal{A}$ (using notation introduced in (34)) are identified. To compare results with those obtained with other methods (cf. sections 3.1, 3.2 and 3.4), the Young's modulus $E$, Poisson coefficient $\nu$ and shear modulus $G$ are locally computed by post-processing the identification results. More elastic constants may be identified, as is the case in section 3.3 where $Q_{x x} Q_{x y} Q_{y y} Q_{s s}$ are sought, if the examined material is homogeneous and the kinematic field is heterogeneous (each finite element yields three independent equations on $Q_{x x} Q_{x y} Q_{y y} Q_{s s}$, and the set of all equations thus obtained is solved in a least-squares sense).

Second variant: enforcement of kinematic measurements by means of a penalty term. It may not necessarily be desirable to enforce exactly the kinematic measurements, especially if they contain measurement noise. For this reason, a variant has been proposed [14] where the functional

$$
\mathcal{F}\left(\boldsymbol{v}, \boldsymbol{\tau}, \mathcal{A}^{\star}\right)=\alpha \mathcal{E}\left(\boldsymbol{v}, \boldsymbol{\tau}, \mathcal{A}^{\star}\right)+\frac{\beta}{2}\|\boldsymbol{v}-\hat{\boldsymbol{u}}\|^{2}
$$

with positive weighting coefficients $\alpha, \beta$ is used instead of $\mathcal{E}\left(\hat{\boldsymbol{u}}, \boldsymbol{\tau}, \mathcal{A}^{\star}\right)$. In (21), the distance $\|\cdot\|$ and the weights $\alpha, \beta$ must be chosen such that the two terms of $\mathcal{F}$ are of similar magnitude. For instance, for a generic displacement $\boldsymbol{w}$, one may set $\|\boldsymbol{w}\|^{2}=E \int_{\Omega} \varepsilon[\boldsymbol{w}]: \varepsilon[\boldsymbol{w}] \mathrm{d} V$ when focussing on the case where $\mathcal{A}$ is isotropic (i.e. defined in terms of e.g. the Young modulus $E$ and the Poisson ratio $\nu$ ), or $\|\boldsymbol{w}\|^{2}=\gamma \int_{\Omega} \boldsymbol{w} \cdot \boldsymbol{w} \mathrm{d} V$ with a coefficient $\gamma$ that has to be properly chosen.

Following other model updating methods based on the CEG [54], the two partial minimizations are performed for different choices of $(\alpha, \beta)$, according to

$$
\mathcal{A}=\underset{\mathcal{A}^{\star} \in \mathbb{A}}{\arg \min } \mathcal{E}\left(\boldsymbol{u}, \boldsymbol{\sigma}, \mathcal{A}^{\star}\right) \quad \text { with } \quad(\boldsymbol{u}, \boldsymbol{\sigma})=\underset{v \in \mathcal{C}(\bar{u}), \tau \in \mathcal{S}}{\arg \min } \mathcal{E}\left(\boldsymbol{v}, \boldsymbol{\tau}, \mathcal{A}^{\star}\right)+\frac{1}{2}\|\boldsymbol{v}-\hat{\boldsymbol{u}}\|^{2}
$$

The stress fields $\tau$ can be represented in the form $\tau=\mathcal{A}^{\star}: \varepsilon[\boldsymbol{w}]$ in terms of displacement fields $\boldsymbol{w}$ defined by a standard finite element discretization, avoiding the recourse to stress finite elements, with the equilibrium constraints in $\mathcal{S}$ enforced in a weak sense corresponding to the chosen finite element basis. As in the first variant, the minimization (22b) with respect to $\mathcal{A}^{\star}$ (correction step) is explicit.

Functionals $\mathcal{F}$ having the form (21) are convex on $\mathcal{C} \times \mathcal{S} \times \mathcal{A}$. As explained in [25,26], another family of functionals $\tilde{\mathcal{F}}(\boldsymbol{v}, \boldsymbol{\tau}, \mathcal{S})$ can be defined from (21) by using the compliance tensor $\mathcal{A}^{-1}$ instead of the rigidity tensor $\mathcal{A}$.

Applications. CEG-based functionals have initially been used for the identification of elastic properties, see e.g $[20,25,26]$. More recently, CEG-based methods have also been proposed and applied in the context of nonlinear constitutive models. Identification of heterogeneous elastoplastic properties and strain energy densities from kinematic field measurements is addressed in [55] and [16], respectively, based on an incremental version of the above-presented elastic CEG where tangent or secant stiffness tensors appearing in incremental (time-discrete) elastic-plastic models are sought. Identification results based on actual experimental data are presented in both references. Other recent 
CEG functionals tailored for nonlinear constitutive parameter identification problems, based on combining the free energy density and its Legendre-conjugate dual potential and potentially applicable to kinematic field measurements, include $[38,70]$. Indeed, CEG functionals can be formulated for all generalized standard materials in terms of the local free energy density and dissipation potential together with their Legendre-conjugate dual potentials (see [67] in the context of error estimation in nonlinear FEM), an avenue not yet fully exploited for material identification purposes to the best of our knowledge.

\subsection{The virtual fields method}

This approach is applicable to situations where the strain field is experimentally known in $\Omega$ :

$$
\varepsilon=\hat{\varepsilon} \quad \text { in } \Omega
$$

where $\hat{\varepsilon}$ is known from full-field measurements (possibly through differentiation of a measured displacement field). No limitations are put on the loading conditions, which are also assumed to be known. One seeks to identify parameters $\boldsymbol{\theta}$ entering an assumed constitutive model for the material, e.g. $A S=\mathcal{A}(\boldsymbol{\theta})$ from overdetermined data in the form of a known strain distribution $\hat{\varepsilon}$. Assuming that no body forces are applied and that boundary conditions are as stipulated in equations $(1,2)$, the principle of virtual work (PVW) takes the form

$$
-\int_{\Omega} \boldsymbol{\sigma}: \boldsymbol{\varepsilon}\left[\boldsymbol{u}^{\star}\right] \mathrm{d} V+\int_{S_{f}} \boldsymbol{T} \cdot \boldsymbol{u}^{\star} \mathrm{d} S=\int_{\Omega} \rho \ddot{\boldsymbol{u}} \cdot \boldsymbol{u}^{\star} \mathrm{d} V \quad \text { for any } \boldsymbol{u}^{\star} \in \mathcal{C}(\mathbf{0})
$$

where $\boldsymbol{u}^{\star}$ is a virtual displacement field.

The virtual fields method (VFM) basically consists of exploiting identity (24) with particular choices of virtual fields [29], tailored to the specific identification problem at hand.

First a constitutive model is chosen, allowing to express $\boldsymbol{\sigma}$ in terms of $\boldsymbol{\theta}$ and the known strain $\hat{\varepsilon}$ in the first integral of (24). For instance, one has $\boldsymbol{\sigma}=\mathcal{A}(\boldsymbol{\theta}): \hat{\varepsilon}$ if elastic moduli are to be identified. It must be emphasized that full-field measurement methods provide measured in-plane strain components over the external surface of solids only. Specimens must therefore be chosen such that strains in the solid can be analytically related to strains on the surface. This is in particular the case for plane stress, plane strain, or bending of thin plates. As a consequence, the first integral can be computed as a function of $\boldsymbol{\theta}$ for any chosen virtual field. Parameter identification for the following classes of constitutive models have been considered in studies carried out during the recent past: linear anisotropic elasticity (in-plane and bending properties of anisotropic plates, either in terms of stiffnesses or invariant combinations of these stiffnesses), nonlinear anisotropic elasticity and, more recently, viscoelasticity and elasto-plasticity. Most of these studies have been carried out in static conditions, for which the third integral of equation (24) vanishes. On a few occasions, e.g. [27, 30], dynamic properties have been identified (hence the inertial term in the r.h.s. of (24)).

Then, virtual fields are chosen. Each virtual field introduced into (24) yields one scalar equation. The constitutive parameters are then sought as solutions to a set of such equations. Obviously, the number of equations thus obtained must be at least equal to the number $M$ of parameters $\theta_{i}$ to be identified. The construction of the virtual fields if a key issue of the method. Three main approaches have been investigated so far:

(i) the easiest one is to construct virtual fields analytically, usually by means of polynomial functions. Such fields are defined over the whole specimen, must fulfill the admissibility condition $\boldsymbol{u}^{\star}=\mathbf{0}$ over $S_{u}$, and are otherwise arbitrary. For instance, in the case $\mathcal{A}(\boldsymbol{\theta})$ of (anisotropic) elasticity, $M$ virtual fields are chosen so that a system of $M$ independent equations governing 
the $M$ parameters $\theta_{i}$ is obtained and solved for $\boldsymbol{\theta}$. In the frequent case of a linear parameterization of the form (9a), the $M$ equations thus obtained define a $M \times M$ invertible linear system of the form (for equilibrium problems)

$$
\sum_{j=1}^{M} C_{i j} \theta_{j}=\int_{S_{f}} \boldsymbol{T} . \boldsymbol{u}_{i}^{\star} \mathrm{d} S \quad \text { with } C_{i j}=\int_{\Omega} \mathcal{A}_{j}: \varepsilon\left[\boldsymbol{u}_{i}^{\star}\right] \mathrm{d} V \quad(1 \leq i \leq M)
$$

When complex-valued moduli are to be determined, as in the case of damping characterization, virtual fields can be complex-valued as well [27].

(ii) A subsequent improvement of the above basic approach (i) consists in a procedure allowing to construct virtual fields automatically [34]. This procedure is applicable only for creating sets of linear equations of the form (25), i.e. in connection with linear parameterizations (9a). Each virtual field $\boldsymbol{u}_{j}^{\star}$ is determined such that $\left[C_{i j}\right]$ is the $M \times M$ identity matrix, i.e. (25) yield directly each $\theta_{i}$ as the virtual work of the external loading in $\boldsymbol{u}_{j}^{\star}$. Such virtual fields are referred to as special. For a given configuration and constitutive model, there are infinitely many such special fields. This extra freedom allows to define special fields so that the identification of $\boldsymbol{\theta}$ is least sensitive to measurement noise [4,34]. The procedure of [4] provides the unique optimal (in that sense) special field which spans a given basis of independent functions chosen a priori.

(iii) the virtual fields can also be defined piecewise instead of having the same functional expression over the whole specimen. Defining subregions in the specimen and constructing virtual fields in each of these subregions allows for more flexibility in their definition. In this case, lower-degree polynomials are used as shape functions in each subregion. Indeed, when virtual fields are defined with the same expression over the whole specimen, higher-degree polynomials may be required and it was observed that such higher-degree polynomials magnify the adverse effect of noise on identified parameters. The only condition here is that the piecewise virtual fields verify the $C^{0}$ continuity between each subregion. It must be emphasized that the $C^{1}$ continuity is not required here: a $C^{1}$ discontinuity does not induce any problem in the calculations, contrary to the similar discontinuity which occurs in the finite element method when actual displacement fields are approximated with piecewise functions.

Three other features concerning the construction of the virtual fields are worthy of emphasis. First, the loading distribution $\overline{\boldsymbol{T}}$, which is involved in the virtual work identity (24), is often difficult to measure in practice. On the other hand, the load resultant is usually measured. In such cases, the virtual fields can be defined so that the virtual work involves only the load resultant. This property is obtained by prescribing a constant virtual displacement over $S_{f}$ along the direction of this load resultant [34]. Second, the virtual work of $\overline{\boldsymbol{T}}$ can be cancelled by selecting virtual fields which vanish on $S_{f}$. This strategy seems to be unavoidable when very local properties are to be identified [83]. Equation (24) then involves only one non-zero integral (the first one) under static conditions. One of the constitutive parameters at least must be considered as known a priori to avoid the final system of linear equations to be homogeneous. If not, then only stiffness ratios can be determined. Third, 3-D integrals must be computed in practice, thus requiring the strain field to be known within the solid. From a theoretical point of view, this feature is undoubtedly a limitation of the VFM. In practice however, relevant assumptions can be used to deduce the displacement/strain fields within the solid from measurements collected on the external surface. For instance, mechanical tests are often carried out on beam- or plate-like specimens. In theses cases, assumptions of constant or linear strain distribution through the thickness of the specimens are sound.

All the strategies described above have been applied in various cases of parameter identification, either with numerical or experimental data. In particular, recent investigations have addressed extensions of the VFM to the identification of material parameters associated with non-linear constitutive 
models. Orthotropic elastic models with damage are considered in [15], while elastic-plastic parameters are identified from sequences of field measurements in $[6,32]$. The corresponding references are summarized in Table 1.

\begin{tabular}{|c|c|c|c|c|c|}
\hline & 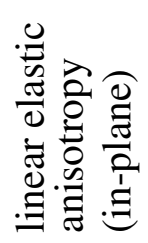 & 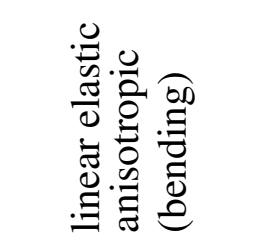 & 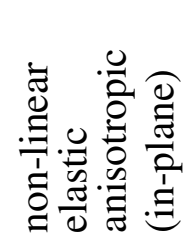 & 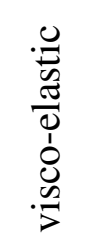 & 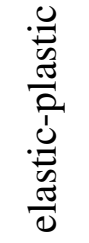 \\
\hline global VF (by hand) & {$[31,33]$} & {$[29,30,36,37]$} & & {$[27]$} & [32] \\
\hline global VF (special) & [34] & {$[35]$} & [34] & - & - \\
\hline piecewise VF & {$[83]$} & - & - & - & [6] \\
\hline
\end{tabular}

Table 1: Types of constitutive equations and virtual field construction procedures used so far in the Virtual Fields Method.

\subsection{The equilibrium gap method}

This approach has been developed for cases where the elastic heterogeneity takes the form of a scalar field $C(\boldsymbol{x})$, i.e.

$$
\mathcal{A}(\boldsymbol{x})=C(\boldsymbol{x}) \mathcal{A}_{0}
$$

(where $\mathcal{A}_{0}$ is given and corresponds to a reference medium) with the aim of identifying this contrast field from the displacement field measured in $\Omega$. For this type of description, the Poisson's ratio is unaffected if an isotropic medium is considered, as in [17]. The scalar contrast $C(\boldsymbol{x})$ may be used to represent isotropic damage $(C(\boldsymbol{x})=1-D(\boldsymbol{x})$, where $0 \leq D(\boldsymbol{x}) \leq 1$ is the damage field [58])

The basic assumption is that the displacement data is available on a measurement grid, which is usually regular. A FEM mesh is then set up so that its nodes coincide with the measurement points. Assuming a constant contrast $C(\boldsymbol{x})=C_{e}$ over each finite element, the stiffness matrix of element $E_{e}$ has the form

$$
\left[\boldsymbol{K}_{e}\right](C)=C_{e}\left[\boldsymbol{K}_{e 0}\right]
$$

where $\left[\boldsymbol{K}_{e 0}\right]$ is the element stiffness matrix for the reference medium endowed with moduli $\mathcal{A}_{0}$. Then, the FEM equilibrium equation at the $m$-th DOF (assumed to be located at a node which does not support any external load) have the form

$$
\sum_{e \mid m \in E_{e}} C_{e}\left\{\boldsymbol{e}_{m}\right\}^{\mathrm{T}}\left[\boldsymbol{K}_{e 0}\right]\left\{\boldsymbol{u}_{e}\right\}=0
$$

where $\left\{\boldsymbol{u}_{e}\right\}$ is the vector of DOFs on element $E_{e},\left\{\boldsymbol{e}_{m}\right\}$ is the $m$-th unit vector, and the summation runs over the elements which share the $m$-th DOF (global numbering is assumed in (28) for expository convenience). Equations (28) provide the basis upon which the contrasts $C_{e}$ are identified, the cofactor of each $C_{e}$ being known by virtue of the assumption that a full-field kinematic measurement is available.

In practice, the set of equations of the form (28) cannot be strictly satisfied (it is usually overdetermined) and a residual force $\boldsymbol{F}_{m}$ arises. Assuming that the set of equations (28) is not underdetermined, the $C_{e}$ can be found e.g. by solving them in a least-squares sense. In that case, positivity constraints on the $C_{e}$ must be enforced explicitly. 
In the special case where DOF number $m$ is shared by exactly two elements $E_{e_{1}}, E_{e_{2}}$ (i.e. is supported by a midside node), equation (28) may be written in logarithmic form as

$$
\ln C_{e_{1}}-\ln C_{e_{2}}=\ln \left|\left\{\boldsymbol{e}_{m}\right\}^{\mathrm{T}}\left[\boldsymbol{K}_{e_{2} 0}\right]\left\{\boldsymbol{u}_{e_{2}}\right\}\right|-\ln \left|\left\{\boldsymbol{e}_{m}\right\}^{\mathrm{T}}\left[\boldsymbol{K}_{e_{1} 0}\right]\left\{\boldsymbol{u}_{e_{1}}\right\}\right|
$$

The change of unknown $c=\ln C$ in (29) allows to satisfy automatically the requirement $C>0$. This formulation, which can only be used for mid-side nodes, has been implemented in [17] for meshes made of eight-noded quadrilateral elements, within a plane-strain or plane-stress framework. For a square mesh made of $N$ elements, the number $K$ of equations (29) is of the order of $4 N$. This redundancy is expected to provide a degree of robustness to the identification scheme.

On defining the vector of transformed unknowns $\{\boldsymbol{c}\}^{\mathrm{T}}=\left\{\ln C_{1}, \ldots, \ln C_{N}\right\}$, the contrast identification problem can now be solved in an unconstrained fashion by means of e.g. a weighted least squares cost function of the form

$$
J\left(\left\{\boldsymbol{c}^{\star}\right\}\right)=\left([\boldsymbol{M}]\left\{\boldsymbol{c}^{\star}\right\}-\{\boldsymbol{q}\}\right)^{t}[\boldsymbol{W}]\left([\boldsymbol{M}]\left\{\boldsymbol{c}^{\star}\right\}-\{\boldsymbol{q}\}\right)
$$

Matrix $[\boldsymbol{M}]$ and vector $\{\boldsymbol{q}\}$ are assembled from the set of equations (29). They depend upon the (measured) nodal displacements. In [17], the weighting $K \times K$ matrix $[\boldsymbol{W}]$ is chosen of the form

$$
\left.[\boldsymbol{W}]=\operatorname{Diag}\left(w_{1}, \ldots, w_{K}\right) \quad \text { with } w_{k}=\mid\left\{\boldsymbol{e}_{k}\right\}^{\mathrm{T}}\left(\left[\boldsymbol{K}_{e_{1, k} 0}\right]\left\{\boldsymbol{u}_{e_{1, k}}\right\}+\boldsymbol{K}_{e_{2, k} 0}\right]\left\{\boldsymbol{u}_{e_{2, k}}\right\}\right)\left.\right|^{\eta} .
$$

with the power $\eta$, adjusted so that the test cases with known contrast distributions lead to the best results, set to $\eta=1.5$.

Upon noting that a uniform distribution over the whole domain amounts to a rescaling of $\mathcal{A}$, to which the measured displacement field is insensitive absent complementary experimental information on applied loads, one of the entries in $\{\boldsymbol{c}\}$ needs to be chosen arbitrarily in order to ensure that $J\left(\left\{\boldsymbol{c}^{\star}\right\}\right)$ has a unique minimizer $\{c\}$.

It must finally be emphasized that the EGM can be considered as equivalent to the VFM employed with some piecewise particular fields, each of them being defined by a non-zero virtual displacement of one of the mid-side nodes of the mesh. For this reason, the EGM suffers from the same limitations as the VFM in terms of data knowledge over $\Omega$ or $\partial \Omega$ (see Section 2.3 above).

\subsection{The Reciprocity Gap Method}

The reciprocity gap method primarily concerns situations where mechanical field measurements are available on the boundary. Let $(\hat{\boldsymbol{u}}, \hat{\boldsymbol{T}})$ denote known displacements and tractions on the boundary. From the virtual work principle, a reciprocity gap functional can then be defined. Consider for the sake of illustration the case where the measurements have been taken on an elastic body $\Omega$ with boundary $S$ endowed with the elasticity tensor $\mathcal{A}(\boldsymbol{x})$, which is to be identified. Besides, let $\boldsymbol{u}^{\star}$ denote the displacement field induced by a traction distribution $T^{\star}$ on $S$ on a body with the same geometry but characterized by the trial elasticity tensor $\mathcal{A}^{\star}(\boldsymbol{x})$ (any such auxiliary state is referred to as an 'adjoint state'). On combining the identities obtained from the virtual work principle applied in turn to the experimental state and the adjoint state (or, equivalently, from the Maxwell-Betti reciprocity theorem for the same two states), one obtains

$$
\int_{\Omega} \varepsilon[\boldsymbol{u}]:\left[\mathcal{A}-\mathcal{A}^{\star}\right]: \varepsilon\left[\boldsymbol{u}^{\star}\right] \mathrm{d} V=\int_{\partial \Omega}\left(\hat{\boldsymbol{T}} \cdot \boldsymbol{u}^{\star}-\boldsymbol{T}^{\star} \cdot \hat{\boldsymbol{u}}\right) \mathrm{d} S \equiv R\left(\mathcal{A}^{\star}, \boldsymbol{u} ; \boldsymbol{u}^{\star}\right)
$$

For any adjoint state $\boldsymbol{u}^{\star}$, the reciprocity gap $R\left(\mathcal{A}, \boldsymbol{u}^{\star}\right)$ is thus a known function of the experimental data $(\hat{\boldsymbol{u}}, \hat{\boldsymbol{T}})$. One must have

$$
R\left(\mathcal{A}^{\star}, \boldsymbol{u} ; \boldsymbol{u}^{\star}\right)=0 \quad \text { for all } \boldsymbol{u}^{\star}
$$


Equation (32) therefore yields an independent scalar relationship on the unknown distribution $\mathcal{A}^{\star}$, or on the parameters involved in its definition, for any choice of adjoint state $\boldsymbol{u}^{\star}$. Note that in general the actual displacement $\boldsymbol{u}$ inside the body is not known a priori and needs to be reconstructed together with $\mathcal{A}$ by means of an iterative procedure. Theoretical studies on identifiability of heterogeneous elastic moduli $[10,47]$ are based on exploiting a linearized version of (31) and with adjoint states chosen according to a procedure initially proposed by Calderon [13], designed so that the right hand side of (31) is the spatial Fourier transform of $\mathcal{A}-\mathcal{A}^{\star}$. These ideas have been extended to the identification of the flexural rigidity tensor in Love-Kirchhoff plates; for instance it is shown in [48] that these rigidities can be uniquely identified from the knowledge of the Dirichlet to Neumann operator, i.e. from the experimental knowledge of all possible pairs $(\hat{\boldsymbol{u}}, \hat{\boldsymbol{T}})$ on the body endowed with $\mathcal{A}^{\star}$. Reciprocity gap functionals are also useful for crack identification, see e.g. [1,7] for equilibrium conditions, and [11] for a variant of this approach, based on a instantaneous reciprocity gap, applicable to crack identification from elastodynamic measurements in the time domain.

The RGM can be considered as a variation on the VFM where kinematical fields are known only on the boundary, when no obvious method for extrapolating this measurement inside the body offers itself. In this form, it has not been applied so far to constitutive identification problems using experimental surface kinematical field data.

\subsection{Summary}

All the above-reviewed identification methods are derived from well-known principles of continuum mechanics, as graphically shown in Figure 1, where the links between the equilibrium equations, the principle of virtual work, the Maxwell-Betti reciprocity principle, the principle of stationarity of the total potential energy and the finite element method are emphasized.

The FEMU and CEGM techniques do not specifically need full-field measurements and can be applied to any kind of overdetermined data. On the other hand, the VFM and EGM rely on the availability of full-field kinematic measurements over the domain. Finally, the RGM is basically tailored so as to use field measurements on the boundary. These requirements are graphically summarized in Figure 1.

Finally, recent developments have shown that when full-field measurements are available and in the case of elasticity, FEMU approaches are equivalent to the VFM where the corresponding virtual fields depend on the choice of the cost function [5].

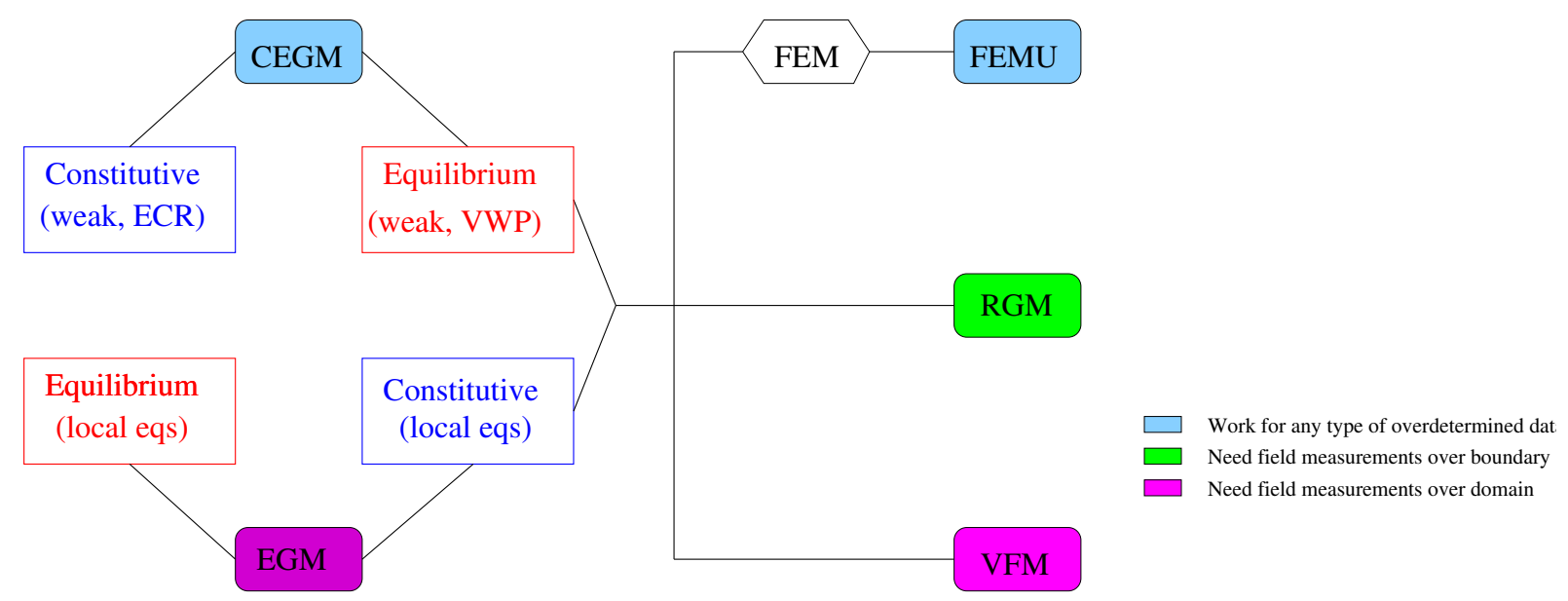

Figure 1: Relationships between the identification strategies and the fundamental principles of continuum mechanics. 


\section{Comparative results obtained using experimental data}

In the following, the identification techniques reviewed in the previous section are applied to fullfield experimental data obtained on four different experiments. Three of these experiments involve non-uniform strain fields in the region of interest. Comparative studies such as those reported in this section, where several identification methods are applied to the same sets of kinematic field data, are very rare in the literature.

1. Tensile test. Ideally, the strain and stress fields associated with such test are uniform. It is therefore not strictly necessary to use full-field measurement techniques and inverse analyses to identify elastic properties. However, considering the fact that a pure tensile test cannot be achieved in practice because of many possible experimental artefacts, and that this test is the one most frequently used to identify elastic parameters, it is instructive to see how inverse analyses perform in this particular case. Moreover, the specific data processed here present high uncertainty levels resulting from the poor quality of the texture used for displacement measurements (Figure 2b).

2. Brazilian test $[59,60]$. An interesting feature of this test is the existence of a closed-form solution that can be established by using either the Airy stress function or, more conveniently for the present purposes, the Kolosov-Muskhelishvili potentials [45] from which the displacement field is directly derived.

3. Shear-flexural test. This test also features a heterogeneous strain field. No closed-form solution is available. Moreover, the tested specimen is orthotropic, and the four in-plane stiffness parameters are involved in the response of the specimen tested using this set-up [15,77]. For identifying simultaneously those four parameters from full-field kinematical measurements acquired during the deformation of the specimen, an inverse approach is required.

4. Biaxial test on a composite material. This test gives rise to a non-uniform damage field, so that at any (sufficiently high) load level the equivalent elastic constitutive properties are nonuniform.

\subsection{Tensile test on an aluminum alloy}

Experimental configuration. A tensile test is carried out on a 2024 aluminum alloy specimen in a servo-hydraulic testing machine. The experimental configuration is shown in Figure 2. Tensile

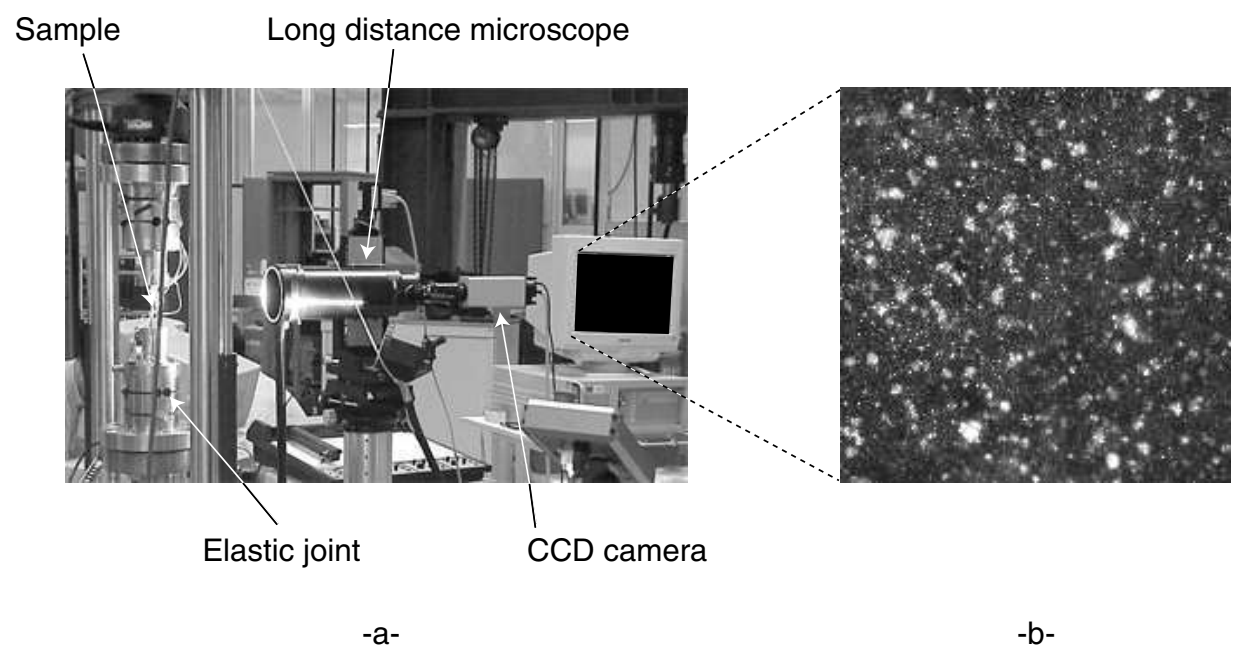

Figure 2: Tensile test: experimental setup for an aluminum alloy sample (a) and reference picture for the DIC analysis (b). 

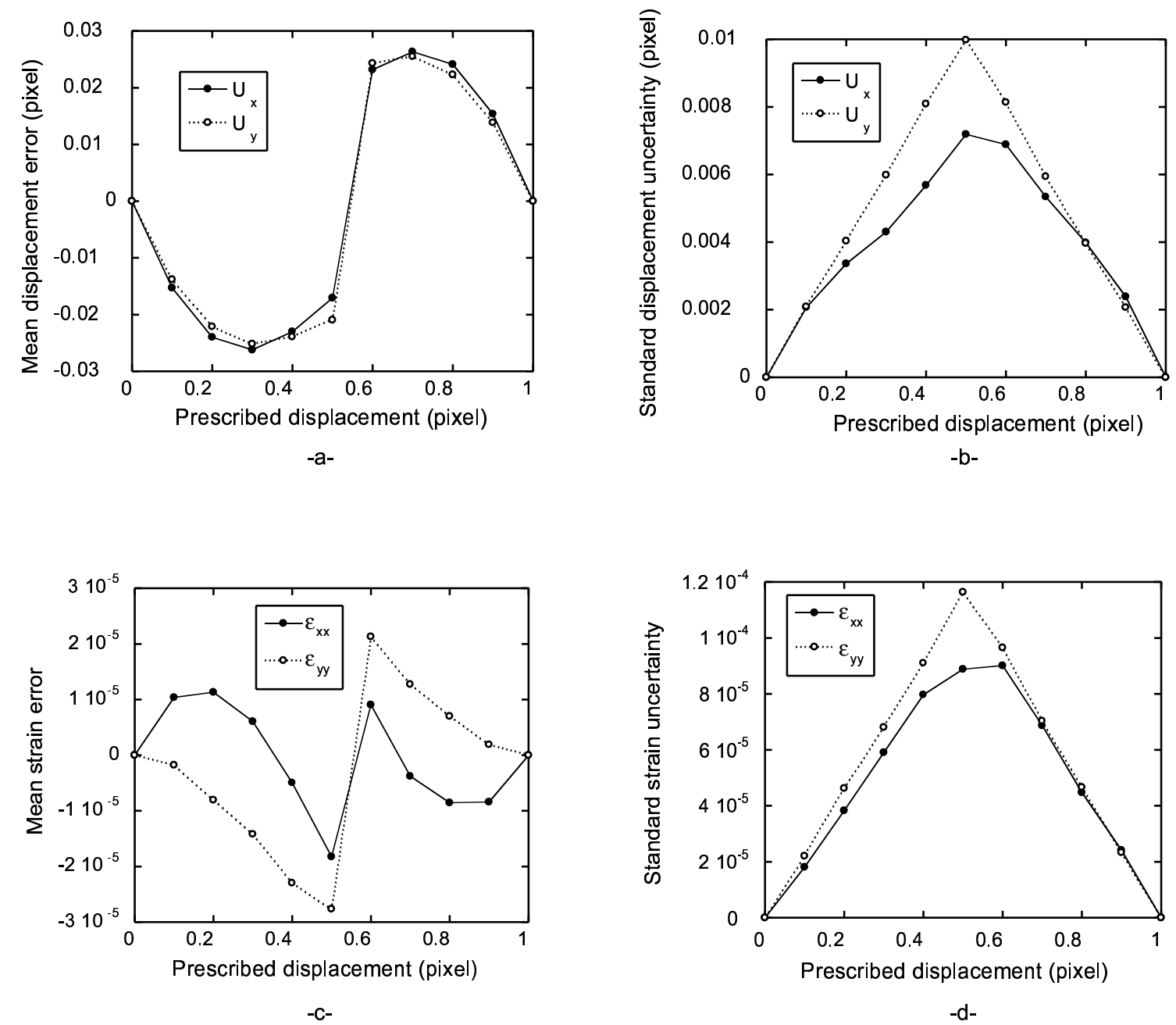

Figure 3: Displacement(a, b) and strain(c, d) errors and standard uncertainties for the picture of Fig. 2-b with a ZOI size of 64 pixels and a shift of 64 pixels when artificially moved by increments of 0.1 pixel.

loads varying between $218 \mathrm{~N}$ and $2003 \mathrm{~N}$ are applied to the sample. A special setup is used to avoid spurious flexure $[40,42]$. The cross-sectional area $S_{0}$ of the gauge section of the sample is equal to $4.8 \times 4.8 \mathrm{~mm}^{2}$. Its geometry is optimized to avoid strain concentrations. Four strain gauges are used to monitor the flexural strains. The average response is used to evaluate the reference strains in the experiment.

Full-field measurement technique. The displacement fields are measured by digital image correlation [41]. The images are taken with an 8-bit CCD camera (resolution: $1008 \times 1016$ pixels). A long-distance microscope monitors a $4 \mathrm{~mm}^{2}$ surface. Each "measurement point" corresponds to the center of an interrogation window (i.e., zone of interest, ZOI) of size $64 \times 64$ pixels. The shift between two neighboring measurement points is equal to 64 pixels. A sub-pixel algorithm is used. It enables a displacement uncertainty of a few hundreds of one pixel for 8-bit pictures $[43,76]$. To achieve a better robustness, an iterative and multi-scale version of the correlation algorithm was used [41, 44]. For each of the 22 applied load levels, a picture is taken by means of the foregoing procedure. The first load level $(218 \mathrm{~N})$ is chosen as the reference state. The image obtained from any of the 21 other load levels provides displacement data that can be used for identification purposes.

The raw data available for identification purposes are displacement fields for 21 load levels, with 225 displacement measurement points used for each load level.

To evaluate the actual performance of the technique, the following steps are performed: 


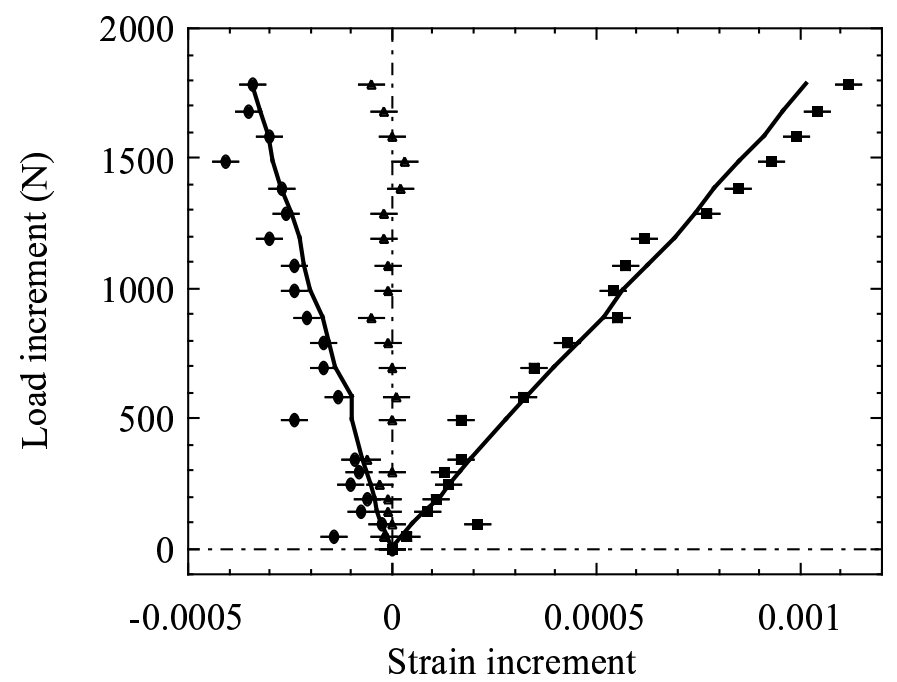

Figure 4: Tensile test: strain gauge measurements (solid lines), average strains obtained from displacement measurements by digital image correlation (symbols).

- A rigid body motion is numerically applied to the reference picture (Fig. 2-b). The algorithm uses FFTs and the shift/modulation property. Ten images are created with displacement increments of 0.1 pixel;

- The eleven-image sequence is analyzed with a DIC software (here CORRELI ${ }^{\mathrm{LMT}}$ [44]) to evaluate the displacements for each image;

- Once all pictures have been analyzed, the mean displacement error and the corresponding standard deviation are determined (Fig. 3). The standard displacement uncertainty is evaluated as the mean of the standard uncertainties for the analyzed sequence. For a given ZOI size, the maximum uncertainty is reached when the prescribed displacement is equal to 0.5 pixel. In this case, the information between each ZOI in the reference and deformed pictures is the most biased;

- For strains, the same eleven pictures are used. It is expected that no strains should be observed since only a rigid body motion is prescribed. The same procedure as for displacements is performed and the mean error and the standard strain uncertainty can be calculated from strain fields as shown in Fig. 3. By using this procedure, the differentiation algorithm yielding the strain field (here based on central finite differences) is tested too. Using another differentiation scheme and another shift would change the strain performance. In terms of uncertainty, the same overall trend as for displacements is observed.

It has to be noted that even if this procedure uses real pictures, all "experimental" noise that exists during an experiment (e.g., lighting, vibrations, texture variations due to strain) is avoided.

Special features. Strain measurements obtained from strain gauges are available. For load levels above $600 \mathrm{~N}$ (i.e., an average stress of $26 \mathrm{MPa}$ ), it is observed that the flexural strains are at most $1 \%$ of the tensile strains [79]. These data are therefore suitable for the evaluation of the elastic properties of the studied material. Figure 4 shows the strain data obtained by the strain gages and by digital image correlation.

One may enquire about the predictive capability of the a priori numerical analysis for evaluating the performance of a correlation algorithm. Figure 5, where the experimental and numerical uncertainties for each ZOI size in both longitudinal and transverse directions are plotted, provides an 


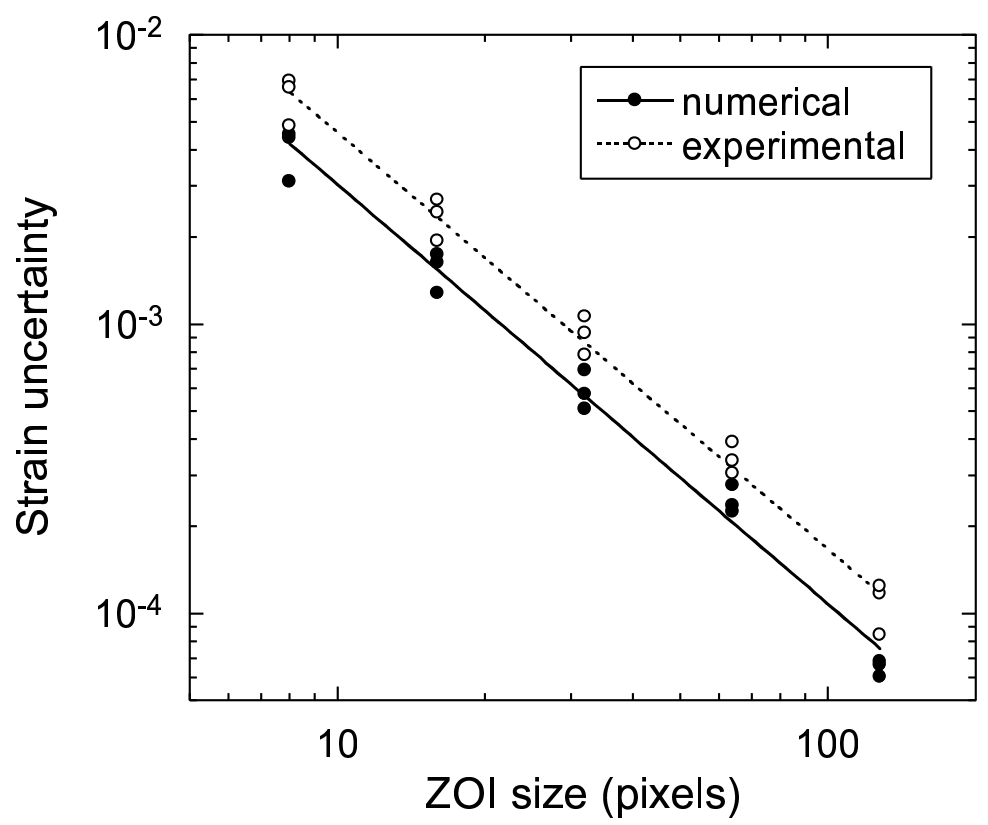

Figure 5: Tensile test: comparison between experimental and numerical strain uncertainties for the picture of Fig. 2-b. The solid and dashed lines are power-law fits of the results.

answer to this question. In a log-log plot a very good fit is obtained with a slope of -1.5 for any strain under scrutiny. There is only a multiplicative offset by a factor of the order of 1.3. Therefore it is concluded that the numerical uncertainty study is an appropriate way of evaluating the overall performance of a DIC algorithm. For the correlation parameters used herein, a strain uncertainty of the order of $3 \times 10^{-4}$ is achieved.

Reference values. The Young's modulus $E$ and Poisson's ratio $\nu$ are determined by using the average strain measurements and the corresponding load levels $F$. On performing a linear regression on these data, the following values of the elastic properties are found: $E_{\text {ref }}=76 \pm 0.5 \mathrm{GPa}$, $\nu_{\text {ref }}=0.33 \pm 0.01$.
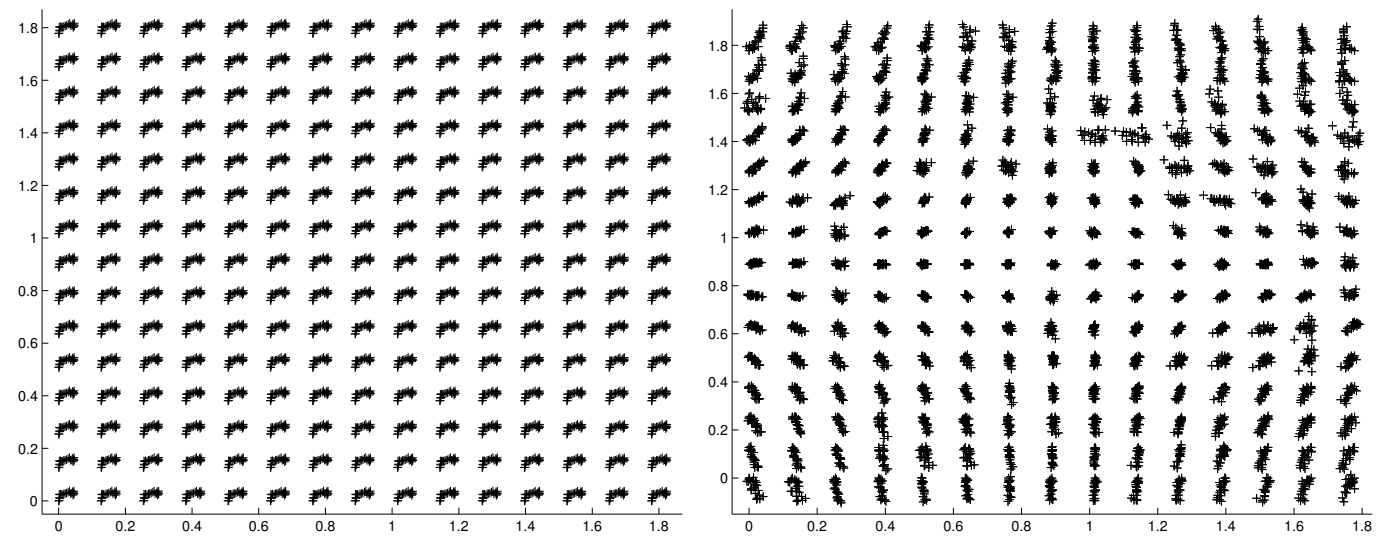

Figure 6: Tensile test: displacement fields measured by DIC (left: raw measurement data; right: measurement data after filtering rigid-body displacements). 
Results. The image correlation data have been processed using several of the techniques presented in Section 2. The elastic parameters actually obtained using each technique are indicated, together with the values of $(E, \nu)$, in Table 2 wherein acronym 'LS' refers to a least-squares estimation based on the exact solution for a homogeneous tensile test with rigid-body motions subtracted out. The notations $Q$ and $Q_{x x}, Q_{y y}$ refer to plane-stress linear-elastic constitutive equations in the $(x, y)$-plane set in the form

$$
\begin{aligned}
\left\{\begin{array}{c}
\sigma_{x} \\
\sigma_{x} \\
\sigma_{s}
\end{array}\right\} & =\left[\begin{array}{ccc}
Q & Q-2 G & 0 \\
Q-2 G & Q & 0 \\
0 & 0 & G
\end{array}\right]\left\{\begin{array}{l}
\varepsilon_{x} \\
\varepsilon_{x} \\
\varepsilon_{s}
\end{array}\right\} \quad \text { (isotropic) } \\
& =\left[\begin{array}{ccc}
Q_{x x} & Q_{x y} & 0 \\
Q_{x y} & Q_{y y} & 0 \\
0 & 0 & Q_{s s}
\end{array}\right]\left\{\begin{array}{l}
\varepsilon_{x} \\
\varepsilon_{x} \\
\varepsilon_{s}
\end{array}\right\} \quad \text { (anisotropic) }
\end{aligned}
$$

in terms of the compressed index notation $x x \rightarrow x, y y \rightarrow y, s s \rightarrow s$.

Table 2 reveals that out of the six methods used, three yield values of $E$ (namely FEMU-U, FEMUU-F and LS) which are quite close to one another, yet their mean value $(\approx 72 \mathrm{GPa})$ differs from $E_{\text {ref }}$ by about $5 \%$. FEMU-F yields the estimate of $E$ closest to $E_{\text {ref. }}$. The largest discrepancy relative to $E_{\text {ref }}$ (about 10\%) occurs for VFM. Such large values are likely to be caused by the high uncertainty levels associated with the quality of the pictures from which the displacement fields are measured. This interpretation is corroborated by the high level of standard uncertainties of the LS technique, indicative of a substantial deviation of the measured displacements from a pure homogeneous tensile response. Concerning the identification of $\nu$, results are seen to exhibit relative scatter higher than those for $E$, as could be expected from the fact that $\nu$ is evaluated as a ratio of strains or moduli, while their mean value $(\approx 0.32)$ is close to $\nu_{\text {ref }}$.

Table 2: Tensile test: chosen parameters and identified values for each technique.

\begin{tabular}{|c||c|c|c|}
\hline Identification technique & parameters & $E(\mathrm{GPa})$ & $\nu$ \\
\hline CEGM1 & $Q_{x x}, Q_{x y}, G$ & $70 \pm 0.9$ & $0.28 \pm 0.01$ \\
\hline FEMU-U & $E, \nu$ & $72.2 \pm 0.4$ & $0.31 \pm 0.01$ \\
\hline FEMU-F & $Q, G$ & $74.4 \pm 0.4$ & $0.36 \pm 0.04$ \\
\hline FEMU-U-F & $E, \nu$ & $71.9 \pm 0.8$ & $0.36 \pm 0.01$ \\
\hline LS & $E, \nu$ & $72 \pm 3$ & $0.3 \pm 0.03$ \\
\hline VFM & $Q, G$ & $69.4 \pm 0.5$ & $0.31 \pm 0.01$ \\
\hline
\end{tabular}

\subsection{Brazilian test}

Experimental configuration. The Brazilian test consists in applying a compressive load along a diameter of a disk-shaped specimen (here made of polycarbonate, with thickness $e=9.87 \mathrm{~mm}$ and diameter $d=155.15 \mathrm{~mm}$ ), as depicted in Figure 7. In contrast with the previous experiment, only one load level $(7470 \mathrm{~N})$ is considered here for the identification.

Full-field measurement technique. The displacement field is measured by digital image correlation. The images are taken with a 12-bit CCD camera (resolution: $1024 \times 1280$ pixels). The same correlation technique as in Section 3.1 is used, and its performance is evaluated in the same way. The 


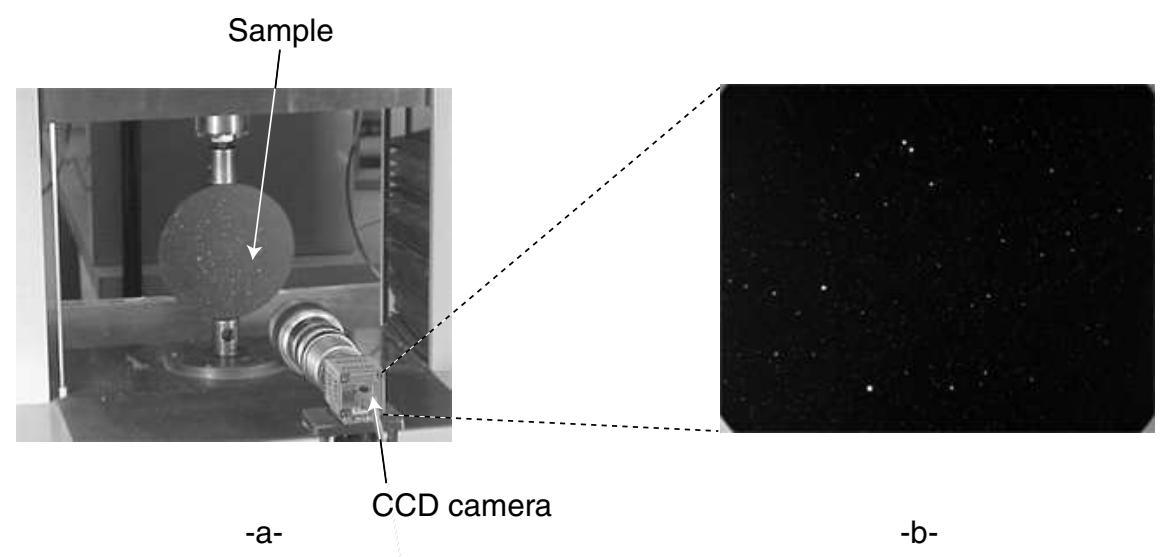

Figure 7: Brazilian test: experimental setup for a polycarbonate sample (a) and reference picture for the DIC analysis (b).
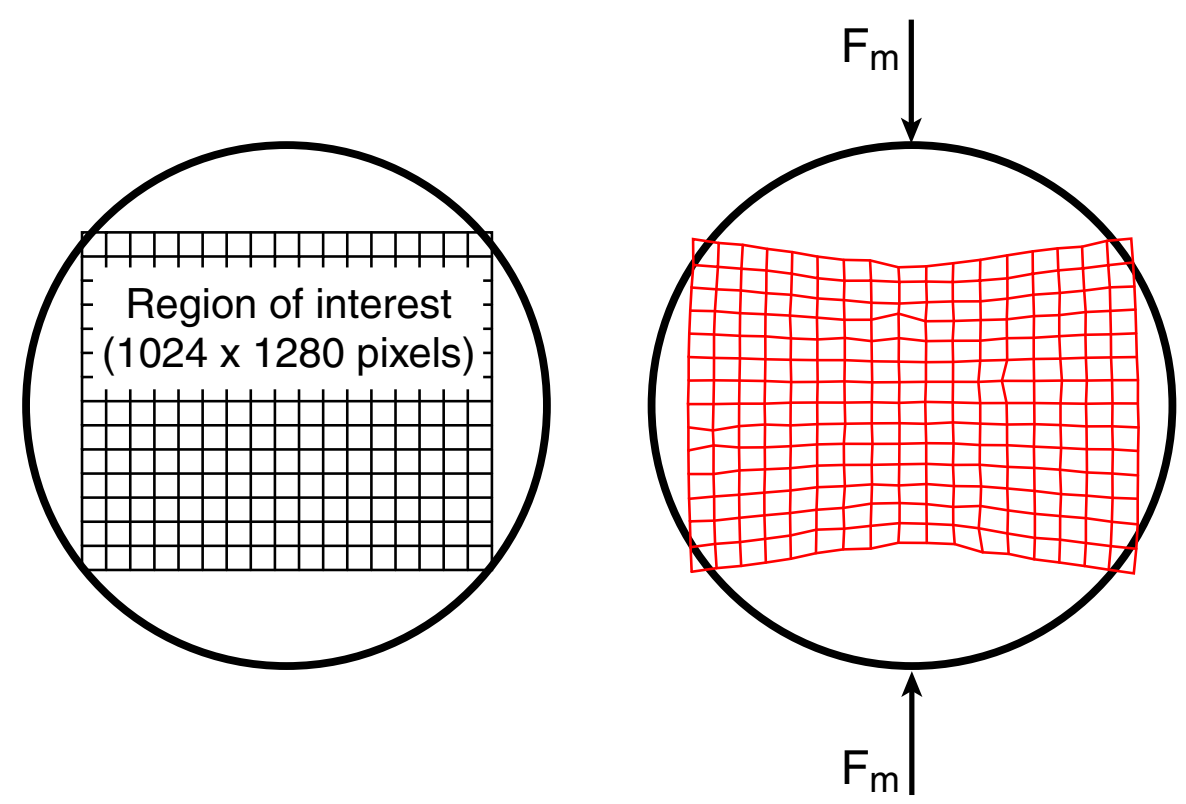

Figure 8: Brazilian test: measured displacement field on the surface of a polycarbonate sample (with a magnification factor of 30).

a priori displacement uncertainty is of the order of $3 \times 10^{-2}$ pixel. This high value is caused by the poor graylevel distribution, as can be seen in Fig. 7-b.

The raw data are made of the displacement field shown in Figure 8. It can be noted that because of the relatively high measurement uncertainty, the displacement field contains measurement points that are clearly unreliable. This is again one of the reasons for choosing this data set.

Reference values. Reference values for Young's modulus $E$ and Poisson's ratio $\nu$ are determined by using the same two pictures, but performing the image correlation in a different way. Specifically, the displacement field is sought in the form of the exact, closed-form, solution to the idealized Brazilian test [45] and the values of $(E, \nu)$ corresponding to the best correlation are retained. The result of this procedure is $E_{\text {ref }}=2.53 \pm 0.02 \mathrm{GPa}, \nu_{\text {ref }}=0.45 \pm 0.01$. Moreover, these reference values have been successfully checked against those obtained by means of a tensile test on the same material [45]. 
Results. The image correlation data have been processed using several of the techniques presented in Section 2. The elastic parameters actually obtained using each technique are indicated in Table 3, together with the values of $(E, \nu)$.

Table 3 shows that all methods except VFM yield values of $E$ which are reasonably close to one another, their mean value $(\approx 2.59 \mathrm{GPa})$ differing from $E_{\text {ref }}$ by about $2.5 \%$. Given the fact that only one load level was used, these results are considered quite satisfactory, and exemplify the benefits brought by using full-field measurements for identification purposes. The performance of the VFM is impaired here by the fact that only $15 \times 15$ available measurement points are available, in contrast with other experiments for which the spatial resolution is much finer, e.g. the shear-flexural test considered next. Values obtained for $\nu$ are greater than $\nu_{\text {ref }}$, and (just barely) consistent with that value in terms of standard uncertainty levels.

Table 3: Brazilian test: chosen parameters and identified values for each technique $(\kappa=(3-\nu) /(1+\nu))$.

\begin{tabular}{|c||c|c|c|}
\hline Identification technique & parameters & $E(\mathrm{GPa})$ & $\nu$ \\
\hline CEGM1 & $Q_{x x} Q_{x y} G$ & $2.59 \pm 0.05$ & $0.47 \pm 0.02$ \\
\hline FEMU-U-F & $E, \nu$ & $2.54 \pm 0.07$ & $0.48 \pm 0.02$ \\
\hline FEMU-U & $E, \nu$ & $2.61 \pm 0.008$ & $0.48 \pm 0.002$ \\
\hline FEMU-F & $Q, G$ & $2.65 \pm 0.21$ & $0.49 \pm 0.06$ \\
\hline LS & $\kappa, G$ & 2.54 & 0.48 \\
\hline VFM & $Q$ & $2.33 \pm 0.05$ & 0.45 (prescribed) \\
\hline
\end{tabular}

\subsection{Shear-Flexural Test}

Experimental configuration. A mechanical test derived from the Iosipescu shear test [49] (see Fig. 10) is carried out for identifying the four in-plane elastic constants of a glass-epoxy unidirectional composite material, the constitutive equations of this material being orthotropic linear elastic. A rectangular-shaped flat specimen is tested in the shear-flexural fixture derived from the Iosipescu set-

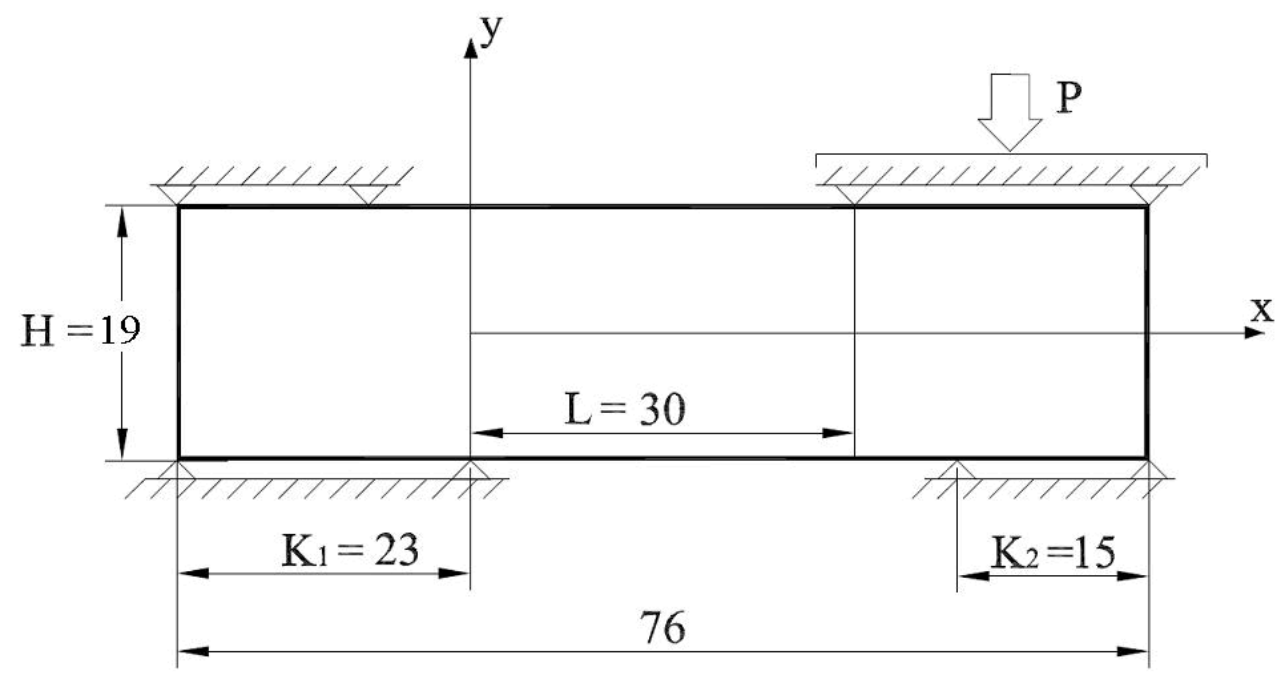

Figure 9: Schematic description of the shear-flexural test (lengths are in millimeters). 


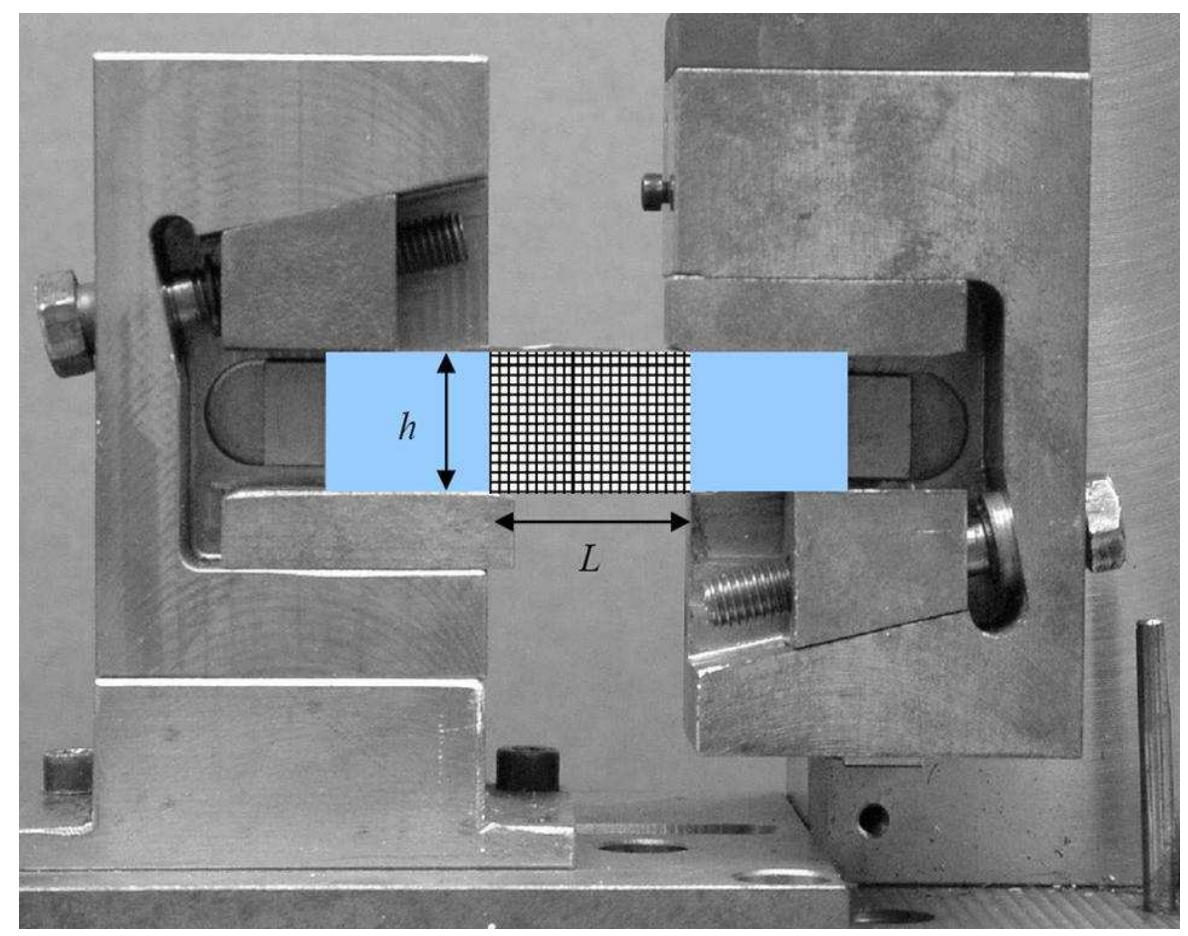

Figure 10: Shear-flexural test fixture with a schematic coupon.

up (see Fig. 9). The thickness $t$ of the specimen is $2.3 \mathrm{~mm}$, the height $h$ is $19 \mathrm{~mm}$, the total length is $76 \mathrm{~mm}$ and the length $L$ of the specimen located in the sheared area is $30 \mathrm{~mm}$. The displacement
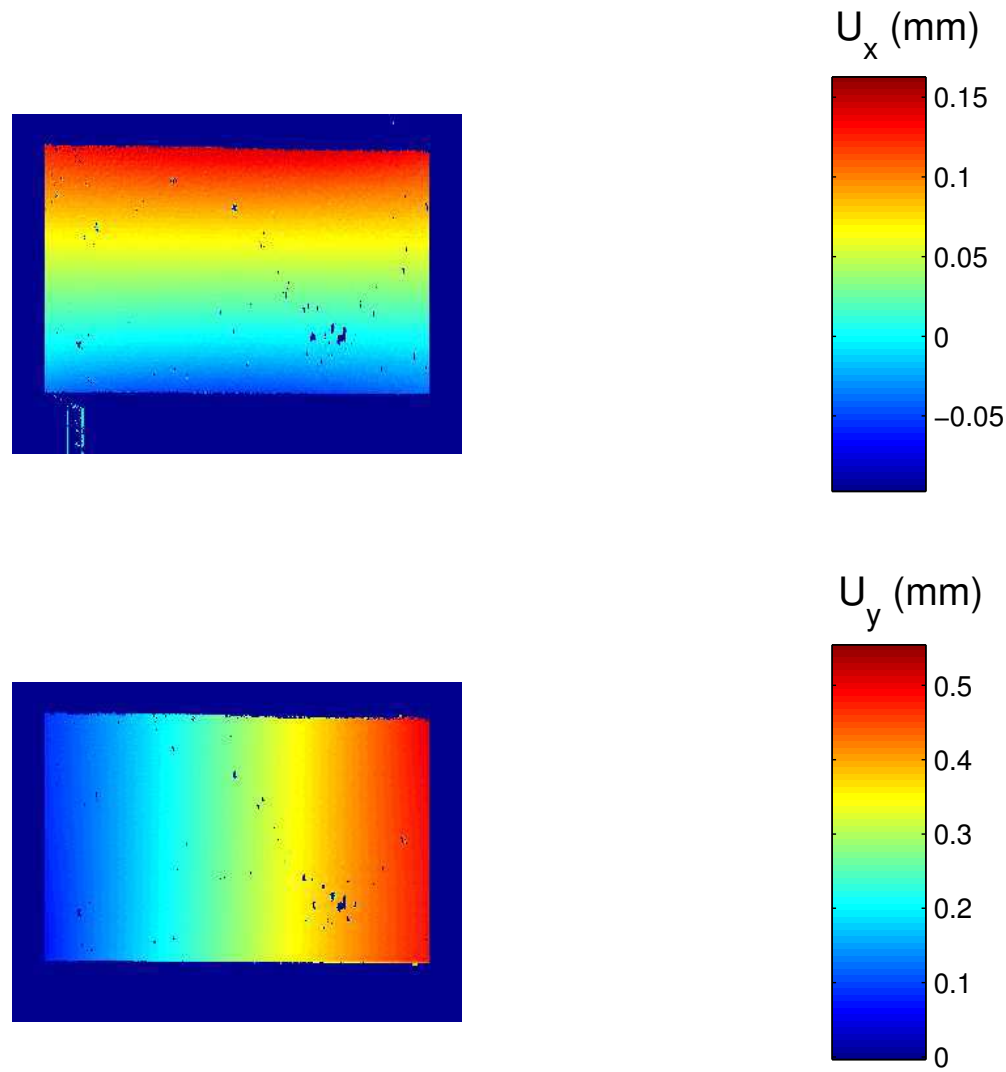

Figure 11: Measured displacement fields in the shear flexural test for an applied load of $600 \mathrm{~N}$. 
fields are measured over the field area (length $L$ in the picture) for an applied load of $600 \mathrm{~N}$ (see Fig. 11). The mechanical behavior of the material remains linear elastic for this applied loading.

Full-field measurement technique. The displacement fields are measured by the grid method. A periodical pattern composed of crossed black lines is transferred onto the surface of the specimen, with a $100 \mu \mathrm{m}$ pitch. The images of the grid are taken with a 12-bit CCD camera (resolution $1024 \times 1280$ pixels). Each image is analyzed as a periodical signal. After deformation of the specimen, phase modulations occur, which are measured by spatial phase shifting with the WDFT algorithm [78]. Finally, both components of displacement are deduced as they are proportional to the phase modulation measured in each direction. This method is rather sensitive to displacements as the resolution evaluated for this experiment is $1.85 \mu \mathrm{m}$, whereas the spatial resolution (equivalent to the size of the correlation window for DIC) is $200 \mu \mathrm{m}$. The resolution is evaluated by measuring two consecutive images of the same grid, by measuring a displacement field between both images and finally by computing the standard deviation of the measured displacement.

Reference values. Standard tests were carried out on the same material to identify reference values for the four in-plane stiffness parameters. Tensile tests along the fibres and perpendicularly to the fibres are used t9o assess $Q_{x x}, Q_{x y}$ and $Q_{y y}$. Standard Iosipescu shear tests (with notched specimens) are used to characterize shear modulus $Q_{s s}$. The average values and coefficients of variations are reported in the table below.

Results. The displacement data have been processed using several of the techniques presented in Section 2. The elastic parameters actually obtained using each technique are indicated in Table 4, along with the reference values of $Q_{x x}, Q_{x y}, Q_{y y}, Q_{s s}$. The identified values of $Q_{s s}$ are very close to one another and to the reference value, consistently with the fact that this test is primarily sensitive to shear.

Table 4: Shear-flexural test: chosen parameters and identified values for each technique.

\begin{tabular}{|c||c|c|c|c|c|}
\hline Identification technique & Parameters & $Q_{x x}(\mathrm{GPa})$ & $Q_{x y}(\mathrm{GPa})$ & $Q_{y y}(\mathrm{GPa})$ & $Q_{s s}(\mathrm{GPa})$ \\
\hline CEGM1 & $Q_{x x}, Q_{x y}, Q_{y y}, Q_{s s}$ & $43.5 \pm 2$ & $3.82 \pm 0.7$ & $11.5 \pm 2.1$ & $3.66 \pm 0.6$ \\
\hline FEMU-U & $Q_{x x}, Q_{x y}, Q_{y y}, Q_{s s}$ & $44.9 \pm 3.1$ & $3.7 \pm 0.8$ & $12.2 \pm 1.6$ & $3.68 \pm 0.7$ \\
\hline VFM & $Q_{x x}, Q_{x y}, Q_{y y}, Q_{s s}$ & $40 \pm 1.1$ & $3.8 \pm 0.3$ & $10.3 \pm 0.4$ & $3.64 \pm 0.1$ \\
\hline reference & $Q_{x x}, Q_{x y}, Q_{y y}, Q_{s s}$ & $44.9 \pm 0.31$ & $3.86 \pm 0.09$ & $12.2 \pm 0.34$ & $3.68 \pm 0.32$ \\
\hline
\end{tabular}

\subsection{Biaxial test on a composite material}

Experimental configuration. The studied material is made of vinylester matrix reinforced by $\mathrm{E}$ glass fibers. A quasi-uniform distribution of fiber orientations leads to an isotropic elastic behavior prior to matrix cracking and fiber breakage, which are the main damage mechanisms [18]. A crossshaped specimen is loaded in a multiaxial testing machine (Fig. 12) in an equibiaxial manner $\left(F_{1}=\right.$ $F_{2}$ ). 


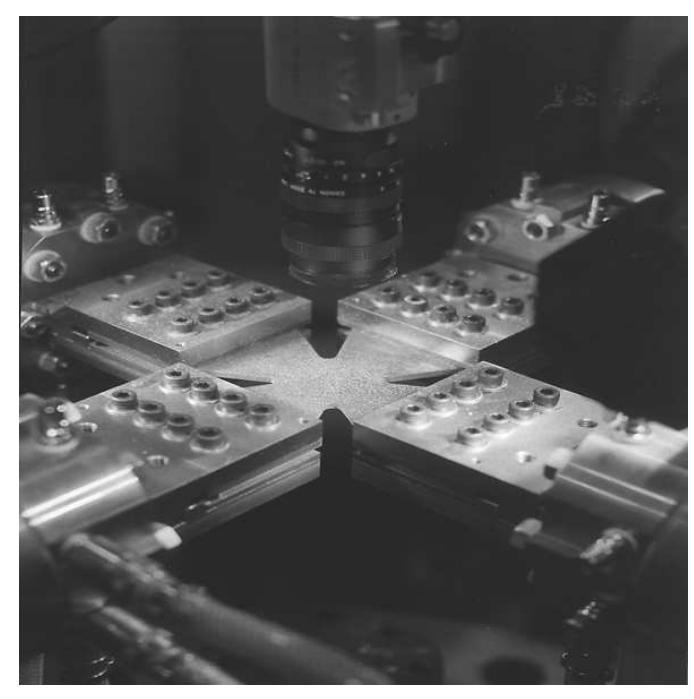

-a-

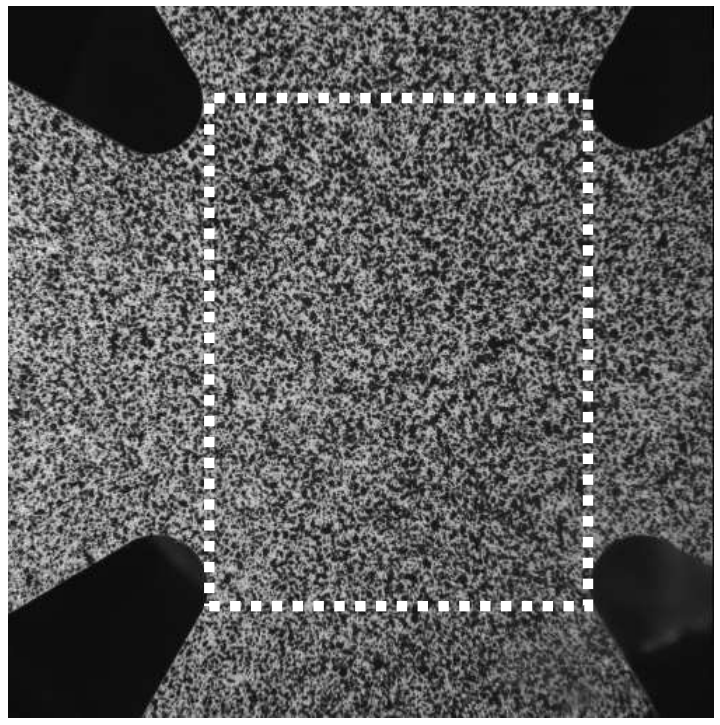

$-b-$

Figure 12: Biaxial test of a composite: experimental setup (a) and reference picture (b) for the DIC analysis. The dashed box depicts the region of interest for the displacement measurement.

Full-field measurement technique. The displacement field is measured by digital image correlation. The images are taken with a 8-bit CCD camera (resolution: $1008 \times 1016$ pixels). The same correlation technique as in Section 3.1 is used.

Data used to evaluate the mechanical properties. The raw data are displacement fields determined for three load levels $\left(F_{1}=F_{2}=5,7,9 \mathrm{kN}\right)$, see Fig. 13.

Special features. The experiment is performed in such a way that the loads applied along two perpendicular directions are of same magnitude. The experiment is controlled in such a way that the specimen center is motionless, thereby avoiding spurious loads. In the present case, three pictures are used for the identification stage. A distinguishing feature of this experiment is that the material

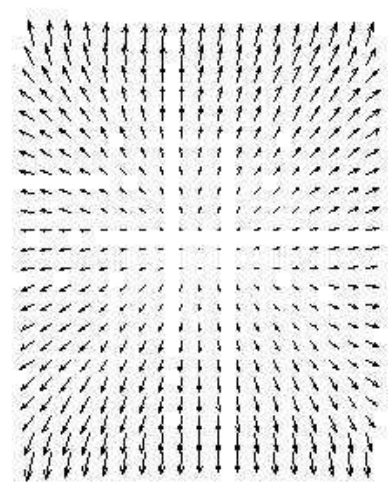

$-a-$

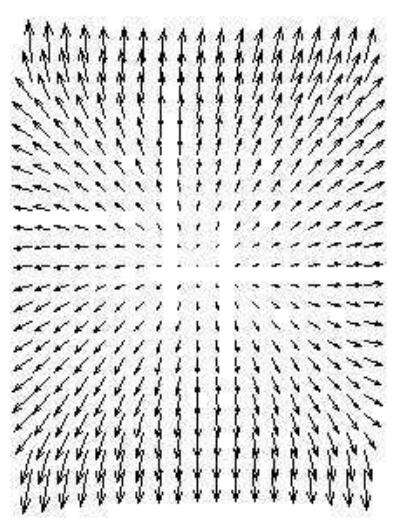

$-b-$

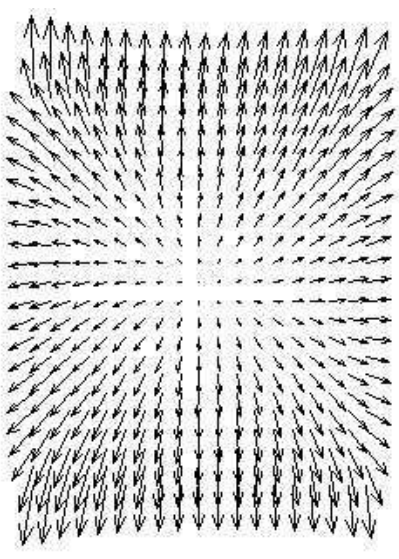

$-\mathrm{C}-$

Figure 13: Biaxial test of a composite: measured displacement fields when $F_{1}=F_{2}=5 \mathrm{kN}$ (a), $7 \mathrm{kN}$ (c) and $9 \mathrm{kN}(\mathrm{c})$, with a magnification factor of 30 . The failure load level is $11.1 \mathrm{kN}$. 


$$
\mathrm{F}=5 \mathrm{kN}
$$

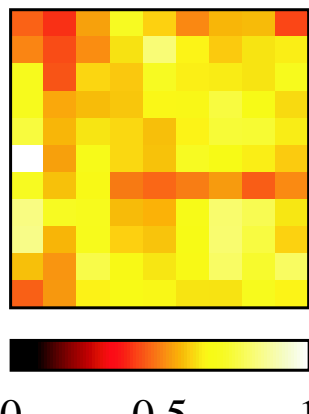

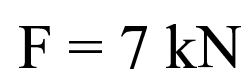

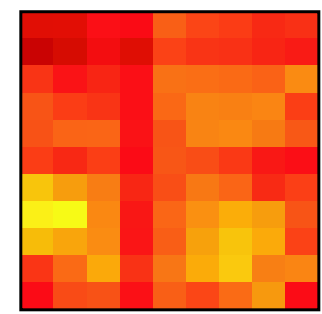

$$
\mathrm{F}=9 \mathrm{kN}
$$

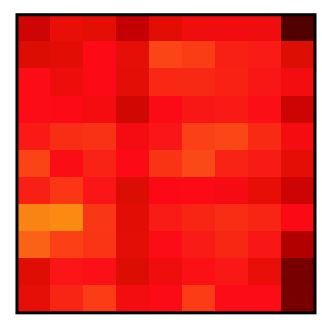

Figure 14: Biaxial test of a composite: distribution of relative Young's modulus $1-D(\boldsymbol{x})$ by damage, for load levels $F_{1}=F_{2}=5 \mathrm{kN}$ (left), $7 \mathrm{kN}$ (middle) and $9 \mathrm{kN}$ (right).

undergoes damage for all three above-indicated load levels.

Reference values. Tensile tests along several directions have been performed on other coupons made of the same material [18]. Quasi-isotropic elastic properties are found, with $E_{\text {ref }}=10 \mathrm{GPa}$ and $\nu_{\text {ref }}=0.28$.

Results. The image correlation data have been processed using several of the techniques presented in Section 2. The elastic parameters actually obtained using each technique are indicated in Table 5, together with the values of $(E, \nu)$. Testing conditions (in particular the fact that $F_{1}=F_{2}$ ) and the choice of region of interest (Fig. 12) made the identification of $\nu$ difficult. For this reason, all treatments (with the exception of FEMU-U and FEMU-UF) were made on the basis of an assumed value of $\nu$. Results obtained for $E$ with the first load level $(5 \mathrm{kN})$ are on average quite consistent with $E_{\text {ref }}$, and are scattered within up to $7 \%$ around that value. Moreover, the diminishing values of $E$ obtained for the two higher load levels using techniques CEGM1-2 are consistent with the fact that damage heterogeneously develops in the sample under increasing loading, see Fig. 14. The EGM method, which is designed for the identification of heterogeneous contrasts of elastic moduli, therefore provides here a means of reconstructing the relative damage field $D(\boldsymbol{x})=1-C(\boldsymbol{x})$ (see Section 2.4).

\section{Conclusion}

Extracting constitutive parameters from full-field measurements is a promising issue because of the recent spread of full-field measurement techniques in the experimental solid mechanics community. This paper presents the main features of five different methods available in the literature for solving this problem, namely the finite element model updating model, the constitutive equation gap method, the virtual fields method, the equilibrium gap method and the reciprocity gap method.

- Updating methods (FEMU-U, FEMU-U-F, CEGM and RGM): in these methods, displacement and/or stress fields are computed numerically, usually by FEM analyses, using an initial guess for the constitutive parameters. Then, the actual parameters are identified by minimizing a cost function where the available measurements are compared to their computed counterparts. The cost function can be defined as the quadratic deviation between the available measures and the computed values at the same location (FEMU-U, FEMU-U-F). Alternatively, it can be defined 
Table 5: Biaxial test on a composite material: chosen parameters and identified values for each technique.

\begin{tabular}{|c||c|c|c|}
\hline Identification technique & Parameters & $E(\mathrm{GPa})$ & $\nu$ \\
\hline CEGM1 $(5 \mathrm{kN})$ & $E$ & 9.8 & 0.28 (prescribed) \\
$(7 \mathrm{kN})$ & & 8.9 & \\
$(9 \mathrm{kN})$ & & 7.9 & \\
\hline CEGM2 $(5 \mathrm{kN})$ & $E$ & 9.3 & 0.28 (prescribed) \\
$(9 \mathrm{kN})$ & & 8.5 & \\
\hline EGM $(5,7,9 \mathrm{kN})$ & & 7.7 & \\
\hline FEMU-U $(5 \mathrm{kN})$ & $E, \nu$ & $9.02 \pm 0.02$ & 0.28 (prescribed) \\
\hline FEMU-F $(5 \mathrm{kN})$ & $Q, G$ & $9.53 \pm 0.92$ & 0.28 (prescribed) \\
\hline FEMU-UF $(5 \mathrm{kN})$ & $E, \nu$ & $9.6 \pm 0.02$ & $0.2 \pm 0.003$ \\
\hline VFM $(5 \mathrm{kN})$ & $Q$ & $9.7 \pm 0.1$ & 0.3 (prescribed) \\
\hline
\end{tabular}

globally (CEGM or RGM) in terms of the deviation between statically and cinematically admissible fields obtained through the numerical computation (CEGM or RGM). For these methods, full-field measurements are useful but not required, which is advantageous in terms of flexibility. They may be defined on the basis of any overdetermined data, e.g. a few measurements distributed across the volume of interest are necessary. On the other hand, such approaches lead to iterative computational procedures, which may on occasion be CPU-intensive, and besides tend to become sensitive to measurement noise if based on scarce experimental data (thus regularization approaches are required).

- Non-updating methods (FEMU-F, EGM and VFM): stress fields are evaluated from measured displacement fields using the postulared constitutive model whose parameters are to be identified, and thus depend implicitly (or explicitly in the case of linear elasticity) upon the latter. Then the equilibrium equations provide the relationships to me solved (implicitly or explicitly) for the sought constitutive parameters. These methods have the advantage of usually leading to fast computational procedures. On the other hand, they specifically require full-field displacement data with sufficiently sharp spatial resolution. Hence the strong current push by experimentalists towards developing and refining experimental techniques providing such field measurements.

Various examples then illustrate the feasibility and the relevancy of these approaches in practice. The experimental data derived from simple mechanical tests: uniaxial and biaxial tensile tests, brazilian tests, shear tests. The data were measured over the surface of the specimens using digital cameras and image processing algorithms. Specimens were thin plates which were assumed to satisfy the plane stress assumption in the field area. Accordingly, only 2D identification was achieved, and focussing only on linear elastic constitutive models.

Some of the obtained results exhibit a substantial scatter about the expected values for the unknown constitutive parameters. Using the same data, two different methods can provide quite different results, especially for the Poisson ratio of the materials tested in the examples. This shows that, even with the large amount of available data, the inverse problem solution remains quite sensitive to error sources in the data. The sensitivity to error sources is higher for non-updating approaches, as they are not amenable to regularization. Non-updating methods may occasionally fail to provide a solution, especially when applied to data with poor spatial resolution. 
The main prospects for future applications of the methods presented in this article concern the identification of constitutive parameters associated with material behavior models that are more complex than the linear elastic case considered here, and for which characterization by classical experimental methods becomes problematic. Elasto-visco-plastic materials under dynamic loading, functionally graded materials..., are examples of such potential applications. The use of full-field measurements for their characterization is promising, but requires substantial progress in either the computational efficiency of updating identification methods, or in the resolution of the experimental imaging technique employed in conjunction with non-updating identification methods.

Other issues remain to be addressed. For instance, the effect of unavoidable noise in the data processed by the different methods should be clearly quantified and minimized by suitable algorithmic procedures. Developing computational sensitivity analysis techniques will be helpful in a posteriori assessment of identification results. Processing heterogenous strain fields also gives an extra freedom which should lead to optimized testing configurations. Several studies are currently underway to tackle these issues in the research groups where the methods reviewed here are developed.

\section{References}

[1] AndrieuX, S., Ben Abda, A., Bui, H. D. Sur l'identification de fissures planes via le concept d'écart à la réciprocité en élasticité. C.R. Acad. Sci. Paris, série II, 324:1431-1438 (1997).

[2] ARAfeH, M. H. Identification de la loi de comportement élastique de matériaux orthotropes. Ph.D. thesis, Université de Technologie de Compiègne (1995).

[3] Arafeh, M.-H., Knopf-Lenoir, C., Rouger, F. Conception optimale d'essais de flexion de plaques orthotropes et identification. Comptes Rendus de l'Académie des Sciences, II/321:351-354 (1995).

[4] Avril, S., Grédiac, M., Pierron, F. Sensitivity of the virtual fields method to noisy data. Computational Mechanics, 34:439-452 (2004).

[5] Avril, S., Pierron, F. General framework for the identification of constitutive parameters from fullfield measurements in linear elasticity. International Journal of Solids and Structures, 44:4978-5002 (2007).

[6] Avril, S., Pierron, F., PAnnier, Y., Rotinat, R. Stress reconstruction and constitutive parameter identification in plane-stress elastoplastic problems using surface measurements of deformation fields. Experimental Mechanics (2008, to appear, DOI 10.1007/s11340-007-9084-2).

[7] Ben Abda, A., Ben Ameur, H., JaOuA, M. Identification of 2D cracks by boundary elastic measurements. Inverse Problems, 15:67-77 (1999).

[8] Bonnet, M. Boundary Integral Equations Methods for Solids and Fluids. John Wiley and Sons (1999).

[9] Bonnet, M., Constantinescu, A. Inverse problems in elasticity. Inverse Problems, 21:R1-R50 (2005). topical review article.

[10] BUI, H. D. Sur quelques problèmes inverses élastiques en mécanique de l'endommagement. In Deuxième Colloque National de Calcul des Structures, pp. 26-35. Hermès, France (1995).

[11] Bui, H. D., Constantinescu, A., Maigre, H. Numerical identification of linear cracks in 2D elastodynamics using the instantaneous reciprocity gap. Inverse Problems, 20:993-1001 (2004).

[12] BurCZyński, T. Evolutionary Computation in Mechanics. IACM Expressions, Bull. for The Int. Assoc. for Comp. Mech. (2003). 
[13] Calderon, A. P. On an inverse boundary value problem. In Seminar on Numerical Analysis and its applications to Continuum Physics., pp. 65-73. Soc. Brasilian de Matematica, Rio de Janeiro (1980).

[14] Calloch, S., Dureisseix, D., Hild, F. Identification de modèles de comportement de matériaux solides: utilisation d'essais et de calculs. Technologies et Formations, 100:36-41 (2002).

[15] Chalal, H., Avril, S., Pierron, F., Meraghni, F. Experimental identification of a damage model for composites using the grid technique coupled to the virtual fields method. Composites: Part A, 37:315325 (2006).

[16] Chrysochoos, A., Berthel, B., Latourte, F., Galtier, A., Pagano, S., Wattrisse, B. Local energy analysis of HCF fatigue using DIC and IRT. The Journal of Strain Analysis for Engineering Design (2008, in press).

[17] Claire, D., Hild, F., Roux, S. A finite element formulation to identify damage fields. Int. J. Num. Meth. in Eng., 61:189-208 (2004).

[18] Collin, F., Berthaud, Y., Hild, F. Visualisation par analyse d'images de la répartition des déformations et de l'amorçage dans un matériau composite. In Y. Berthaud, M. Cottron, F. Dupré, M. Morestin, P. Moucheront, M. Taroni (eds.), Photomécanique 1998, pp. 241-248. GAMAC (1998).

[19] Collins, J.C., Hart, G.C., Kennedy, B. Statistical identification of structures. AiAA Journal, 12:185-190 (1974).

[20] Constantinescu, A. On the identification of elastic moduli from displacement-force boundary measurements. Inverse Problems in Engineering, 1:293-315 (1995).

[21] Cottin, N., Felgenhauer, H.P., Natke, H.G. On the parameter identification of elastomechanical systems using input and ouput residuals. Ingenieur-Archiv, 54:378-387 (1984).

[22] Cugnoni, J., Gmur, T., Schorderet, A. Inverse method based on modal analysis for characterizing the constitutive properties of thick composite plates. Computers and Structures, 85:1310-1320 (2007).

[23] Furgiuele, F.M., Muzzupappa, M., Pagnotta, L. A full-field procedure for evaluating the elastic properties of advanced ceramics. Experimental Mechanics, 37:285-291 (1997).

[24] Genovese, K., Lamberti, L., C., Pappalettere. Mechanical characterization of hyperelastic materials with fringe projection and optimization techniques. Optics and Lasers in Engineering, 44:423-442 (2006).

[25] Geymonat, G., Hild, F., Pagano, S. Identification of Elastic Parameters by Displacement Field Measurement. C.R. Acad. Sci. Paris, série II, 330:403-408 (2002).

[26] Geymonat, G., PAGano, S. Identification of mechanical properties by displacement field measurement: a variational approach. Meccanica, 38:535-545 (2003).

[27] Giraudeau, A., Pierron, F. Simultaneous identification of stiffness and damping properties of isotropic materials from forced vibrating plates. Comptes Rendus Mécanique, 331:259-264 (2003).

[28] Giton, M., Caro-Bretelle, A.S., Ienny, P. Hyper-Elastic Behaviour Identification by a Forward Problem Resolution : application to a tear test of a Silicone-Rubber. Strain, 42:291-297 (2006).

[29] GrÉDIAC, M. Principe des travaux virtuels et identification. Comptes Rendus de l'Académie des Sciences, II/309:1-5 (1989).

[30] Grédiac, M., Fournier, N., Paris, P.-A., Surrel, Y. Direct identification of elastic constants of anisotropic plates by modal analysis : experiments and results. Journal of Sound and Vibration, 210:645659 (1998). 
[31] Grédiac, M., Pierron, F. A T-shaped specimen for the direct characterization of orthotropic materials. International Journal for Numerical Methods in Engineering, 41:293-309 (1998).

[32] Grédiac, M., Pierron, F. Applying the Virtual Fields Method to the identification of elasto-plastic constitutive parameters. International Journal of Plasticity, 22:602-627 (2006).

[33] Grédiac, M., Pierron, F., Y., Surrel. Novel procedure for complete in-plane composite characterization using a T-shaped specimen. Experimental Mechanics, 39:142-149 (1999).

[34] Grédiac, M., Toussaint, E., Pierron, F. Special virtual fields for the direct determination of material parameters with the virtual fields method. 2- Application to in-plane properties. International Journal of Solids and Structures, 39:2707-2730 (2002).

[35] Grédiac, M., Toussaint, E., Pierron, F. Special virtual fields for the direct determination of material parameters with the virtual fields method. 3- Application to the bending rigidities of anisotropic plates. International Journal of Solids and Structures, 40:2401-2419 (2003).

[36] Grédiac, M., Vautrin, A. A new method for determination of bending rigidities of thin anisotropic plates. Journal of Applied Mechanics, 57:964-968 (1990).

[37] GRÉDiaC, M., VAutRin, A. Mechanical characterization of anisotropic plates : experiments and results. European Journal of Mechanics/A Solids, 12:819-838 (1993).

[38] Hadj-Sassi, K., Andrieux, S. Une nouvelle fonctionnelle d'énergie incrémentale totale pour le contrle des parties réversibles et dissipatives des matériaux standards. In Actes du Huitième Colloque National en Calcul des Structures, pp. 255-261. Hermes (2007).

[39] Hemez, F.M., FARHAT, C. Updating finite element dynamic models using element-by-element sensitivity methodology. AIAA Journal, 32:1702-1711 (1993).

[40] HiLD, F. Dispositif de traction-compression d'une éprouvette. E.N.S. Cachan/Renault, French patent nr. 9006848 (Bulletin Officiel de la propriété industrielle, 92/33, publication nbr. 2662 801) (1992).

[41] HiLD, F. CORRELI ${ }^{\mathrm{LMT}}$ : A Software for Displacement Field Measurements by Digital Image Correlation. LMT-Cachan, internal report 254 (2002).

[42] Hild, F., Amar, E., Marquis, D. Stress Heterogeneity Effect on the Strength of Silicon Nitride. J. Am. Ceram. Soc., 75:700-702 (1992).

[43] Hild, F., Périé, J.-N., Coret, M. Mesure de champs de déplacements 2D par intercorrélation d'images :CORRELI ${ }^{\mathrm{LMT}}$. LMT-Cachan, internal report 230 (1999).

[44] Hild, F., Raka, B., Baudequin, M., Roux, S., Cantelaube, F. Multi-scale displacement field measurements of compressed mineral wool samples by digital image correlation. Appl. Optics, IP 41:6815-6828 (2002).

[45] Hild, F., Roux, S. Digital image correlation: from measurement to identification of elastic properties A review. Strain, 42:69-80 (2006).

[46] Hoc, T., GÉlébart, L., Crépin, J., Zaoui, A. A procedure for identifying the plastic behaviour of single crystals from the local response of polycrystals. Acta Materialia, 51:5479-5490 (2003).

[47] IKEHATA, M. Inversion formulas for the linearized problem for an inverse boundary value problem in elastic prospection. SIAM J. Appl. Math., 50:1635-1644 (1990).

[48] Ikehata, M. An inverse problem for the plate in the Love-Kirchhoff Theory. SIAM J. Appl. Math., 53:942-970 (1993).

[49] Iosipescu, N. New accurate procedure for single shear testing of metals. J. Mater, 2:537-566 (1967). 
[50] KAJBerg, J., LindkVist, G. Characterization of materials subjected to large strains by inverse modelling based on in-plane displacement fields. International Journal of Solids and Structures, 41:34393459 (2004).

[51] Kajberg, J., Sundin, K.G., Melin, L.G., StÅhle, P. High strain rate tensile and viscoplastic parameter identification using micoscopic high-speed photography. International Journal of Plasticity, 20:561-575 (2004).

[52] Kavanagh, K.T., Clough, R.W. Finite element applications in the characterization of elastic solids. International Journal of Solids and Structures, 7:11-23 (1971).

[53] Ladevèze, P., Nedjar, B., Reynier, M. Updating of Finite Element Models Using Vibration Tests. AIAA, 32:1485-1491 (1994).

[54] Ladevèze, P., Reynier, M. A Localization Method of Stiffness Errors for the Adjustement of F.E. Models. In Proceedings F.E. Modeling and Analysis in Vibrations Analysis Techniques and Applications, pp. 355-361. ASME (1989).

[55] Latourte, F., Chrysochoos, A., Pagano, S., Wattrisse, B. Elastoplastic behavior identification for heterogeneous loadings and materials. Experimental Mechanics (2008, in press).

[56] Lecompte, D., Sol, H., Vantomme, J., Habraken, A.M. Identification of Elastic Orthotropic Material Parameters Based on ESPI Measurements. In SEM Annual Conference and Exposition on Experimental and Applied Mechanics (2005).

[57] Lecompte, D., Sol, H., Vantomme, J., Habraken, A.M. Mixed numericl-experimental technique for orthotropic parameter identification using biaxial tensile tests on cruciform specimens. Int. J. Solids Struct., 44:1643-1656 (2007).

[58] Lemaître, J., Chaboche, J. L. Mechanics of solid materials. Cambridge University Press (1990).

[59] Lobo Carneiro, F. C. Um novo método para determinacão da resistência à tração dos concretos. Proceedings Anais 5a reunião da Associação Brasileira de Normas Técnicas (ABNT) em São Paulo, 127129 (1943).

[60] Lobo CARneiro, F. C. Une nouvelle méthode pour la détermination de la résistance à la traction des bétons. Bull. RILEM 13:103-108 (1953).

[61] Magorou, L. Le, Bos, F., Rouger, F. Identification of constitutive laws for wood-based panels by means of an inverse method. Composite Science and Technology, 62:591-596 (2002).

[62] Mahnken, R. A comprehensive study of a multiplicative elastoplasticity model coupled to damage to include parameter identification. Computers and Structures, 74:179-200 (2004).

[63] Mauvoisin, G., Bremand, F. J., Lagarde, A. Three-dimensional shape reconstruction by phaseshifting shadow moire. Applied Optics, 33:2163-2169 (1994).

[64] Meijer, R., Douven, L.F.A., Oomens, C.W.J. Characterisation of anisotropic and non-linear behaviour of human skin in-vivo. Comput. Methods in Biomechanics and Biomedical Engineering, 1:13-27 (1997).

[65] Meuwissen, M. An inverse method for the mechanical characterization of metals. Ph.D. thesis, Eindhoven University of Technology (1998).

[66] Meumissen, M. H. H., Oomens, C. W. J., BaAijens, F. P. T., Petterson, R., Janssen, J. D. Determination of the elasto-plastic properties of aluminium using a mixed numerical-experimental method. Journal of Materials Processing Technology, 75:204-211 (1998). 
[67] MoËs, N., LAdevèze, P., Douchin, B. Constitutive relation error estimators for (visco)plastic finite element analysis with softening. Comp. Meth. in Appl. Mech. Engng., 176:247-264 (1999).

[68] Molimard, J., Le Riche, R., Vautrin, A., Lee, J.R. Identification of the four orthotropic plate stiffnesses using a single open-hole tensile test. Experimental Mechanics, 45:404-411 (2005).

[69] Moreau, A., Pagnacco, E., Borza, D., Lemosse, D. An evaluation of different mixed experimental/numerical procedures using FRF for the identification of viscoelastic materials. In International Conference on Noise and Vibration Engineering, ISMA 2006, Leuven (2006).

[70] Nguyen, H. M., Allix, O., Feissel, P. Application of the CRE for parameter identification in nonlinear dynamics with corrupted measurements. Seventh World Congress on Computational Mechanics, Los Angeles, USA (2006).

[71] Oomens, C.W.J., van Ratingen, M.R., Janssen, J.D., KoK, J.J., Hendriks, M.A.N. A numerical-experimental method for a mechanical characterization of biological materials. Journal of Biomechanics, 26:617-621 (1993).

[72] Padmanabhan, S., Hubner, J.P., Kumar, A.V., Ifuu, P.G. Load and Boundary Condition Calibration Using Full-field Measurement. vol. 46, pp. 569-578 (2006).

[73] Pagnacco, E., Lemosse, D. A coupled FE based inverse strategy from displacement field measurement subject to an unknown distribution of forces. In Photomechanics 2006, Clermont-Ferrand, France (2006).

[74] Pagnacco, E., Lemosse, D., Hild, F., Amiot, F. Inverse strategy from displacement field measurement and distributed forces using FEA. In 2005 SEM Annual Conference and Exposition on Experimental and Applied Mechanics (2005).

[75] Pagnacco, E., Moreau, A., Lemosse, D. Inverse strategies for the identification of elastic and viscoelastic material paremeters using full-field measurements. Materials Science and Engineering: A, 452-453:737-745 (2007).

[76] Perié, J. N., Calloch, S., Cluzel, C., Hild, F. Analysis of a multiaxial test on a C/C composite by using digital image correlation and a damage model. Experimental Mechanics, 42:318-328 (2002).

[77] Pierron, F., Grédiac, M. Identification of the through-thickness moduli of thick composites from whole-field measurements using the Iosipescu fixture: theory and simulations. Composites: Part A, 31:309-318 (2000).

[78] Rastogi, P.K. Photomechanics. Springer Verlag (1999).

[79] Renaud, P. Comportement d'un matériau composite carbone/carbone 3D sous chargement uniaxial. Master's thesis, ENS Cachan (2000).

[80] SurRel, Y. Les techniques optiques de mesure de champ: essai de classification. Instrumentation, mesure et Métrologie, 4:11-42 (2004).

[81] TARAntola, A. Inverse problem theory. Elsevier (1987).

[82] Thomas, J. W. Numerical partial differential equations: conservation laws and elliptic equations. Springer-Verlag (1999).

[83] Toussaint, E., Grédiac, M., Pierron, F. The virtual Fields method with piecewise virtual fields. International Journal of Mechanical Sciences, 48:256-264 (2006).

[84] VAN RATINGEN, M.R. Mechanical identification of inhomogeneous solids: a mixed numerical experimental approach. Ph.D. thesis, Eindhoven University of Technology (1994). 